\title{
A Simulation Study on Latent Transition Analysis for Examining Profiles and Trajectories in Education: Recommendations for Fit Statistics
}

Peter A. Edelsbrunner ${ }^{1 *}$; Maja Flaig ${ }^{2 *}$, Michael Schneider ${ }^{3}$

${ }^{1}$ ETH Zurich

${ }^{2}$ University of Tuebingen

${ }^{3}$ University of Trier

This is an author preprint, please cite as:

Edelsbrunner, P. A., Flaig, F., \& Schneider, M. (in press). A simulation study on latent transition analysis for examining profiles and trajectories in education: Recommendations for fit statistics. Journal of Research on Educational Effectiveness.

Author note: *Authors contributed equally. Corresponding author: Peter Edelsbrunner; Emailaddress: peter.edelsbrunner@ifv.gess.ethz.ch; Postal address: ETH Zurich, Clausiusstrasse 59, 8092 Zurich, Switzerland.

Acknowledgments:

We thank Simon Grund, Oliver Lüdtke, Gabriel Nagy, Alexander Robitzsch, and Jeroen Vermunt, as well as the organizers and attendees of the First Symposium on Classification Methods in the Social and Behavioral Sciences hosted at Tilburg University, for informative discussions and helpful feedback. Furthermore, we thank Fabian Dablander for help with descriptions of technical details. 


\begin{abstract}
Latent transition analysis is an informative statistical tool for depicting heterogeneity in learning as latent profiles. We present a Monte Carlo smulation study to guide researchers in selecting fit indices for identifying the correct number of profiles. We simulated data representing profiles of learners within a typical pre- post- follow up-design with continuous indicators, varying sample size ( $N$ from 50 to 1000 ), attrition rate (none/10\% per wave), and profile separation (entropy; from .73 to .87$)$. Results indicate that the most commonly used fit index, the Bayesian information criterion $(B I C)$, and the consistent Akaike information criterion $(C A I C)$ consistently underestimate the real number of profiles. A combination of the $A I C$ or the $A I C 3$ with the adjusted Bayesian Information Criterion $(a B I C)$ provides the most precise choice for selecting the number of profiles and is accurate with sample sizes of at least $N=200$. The $A I C 3$ excels starting from $N=500$. Results were mostly robust towards differing numbers of time points, profiles, indicator variables, and alternative profiles. We provide an online tool for computing these fit indices and discuss implications for research.
\end{abstract}

Keywords: Latent transition analysis; learning patterns; education; information criteria; simulation study 


\section{A Simulation Study on Latent Transition Analysis for Examining Profiles, Patterns, and Trajectories in Education: Recommendations for Fit Statistics}

Educational researchers are often interested in studying heterogeneity in learning. For whom and under what conditions does an educational intervention work? How do learners differ from each other on central learning outcomes? How do they differ concerning their learning trajectories? Reasons for heterogeneity in learning are manifold and include characteristics of learners as well as characteristics of the learning context, such as the class, teacher, school, or home (e.g., Hattie, 2009; Brühwiler \& Blatchford, 2011). Usually not all covariates that determine individual differences in learning are known. This means that there is unobserved heterogeneity, such that we do not have the information available to identify differences in learning patterns. When this is the case, it remains unstudied for whom "average" patterns of learning across a whole population are representative, leaving important aspects of heterogeneity in learning unobserved. For modeling such unobserved heterogeneity, person-centered statistical approaches are commonly used (Harring \& Hodis, 2016). In these approaches, instead of correlating different variables across all learners, the learners are assigned to sub-groups based on their observed patterns on outcome variables. These sub-groups, called classes or profiles, capture individual differences of the learning process.

In the present research, we conduct a simulation study to examine the adequacy of different fit indices for the person-centered, longitudinal approach of latent transition analysis with continuous indicator variables (LTA; Hickendorff et al., 2018). In an LTA with continuous indicators, learners are grouped into latent profiles based on their patterns of means and variances across multiple variables that capture aspects of the learning process (e.g., achievement, knowledge, or motivation). In addition, capitalizing on longitudinal information, 
the probability that the learners transition (i.e., switch) between different profiles over time is modeled. In educational research, these transitions would typically represent learning. A difficult aspect in the specification of these models is that the user has to decide how many latent profiles of learners should be modelled (Hickendorff et al., 2018). Usually, this decision is based on different fit statistics. In the present study, we conduct a simulation study to examine which fit statistics work best for selecting the correct number of profiles in a latent transition analysis.

\section{Person-Centered Approaches to Studying Heterogeneity Among Learners}

Whereas variable-centered approaches focus on examining links between learner or context characteristics and learning outcomes, person-centered approaches aim at grouping learners according to distinct learning characteristics. Person-centered approaches have been proven useful tools to describe how learners differ at a single time point or in their learning trajectories across multiple time points (Hickendorff et al., 2018). LTA is one informative statistical tool for examining these questions that has been recently established in educational research. In this type of person-centered analysis, learners are clustered into homogenous subgroups according to their measured values on multiple variables that are meant to capture the learning process. LTA has been used for example to study the structure and development of children's knowledge about density and buoyancy force (Edelsbrunner et al., 2015, 2018; Schneider \& Hardy, 2013), psychology students' understanding of human memory (Flaig et al., 2018), secondary school students' understanding of rational numbers (Kainulainen et al., 2016), and motivational development across multiple subjects (Costache et al., 2021; Franzen et al., 2022). Cross-sectional versions of person-centered approaches have been used for example to identify learners at risk of developing reading disabilities (Swanson, 2012), low literacy (Mellard et al., 2016), motivational patterns across subjects (Gaspard et al., 2019), patterns of competence 
levels across multiple skills in science (Schwichow et al., 2020), or maladaptive learning strategies (Fryer, 2017).

\section{Fit Indices for LTA}

A central question in LTA concerns the number of profiles or clusters in the final model. For example, is a two-class model superior to a three-class model to describe literacy profiles in a sample of adolescent and young adult learners? One helpful guide in estimating the number of expected profiles or clusters in a sample is educational theory. However, theory alone is usually not sufficient for deciding on the number of expected profiles. In that case, statistical indices are required to help to decide on the number of profiles. For example, when studying profiles of conceptual knowledge, it may be theoretically sound to extract two profiles (one defined by many misconceptions, and one scientific profile; Edelsbrunner et al., 2018; Schneider \& Hardy, 2013), three profiles (misconceptions, fragmented knowledge, and scientific profile), four profiles (misconceptions, fragmented knowledge, indecisive profile, and prescientific profile), or even more depending on the theoretical framework and the measurement model. Researchers need additional criteria to decide which model is best suited to characterize learners in their sample. Model fit indices, that is, statistical criteria to decide on the best fitting model regarding number of profiles/clusters are an important tool in this regard.

Typical statistical comparisons such as likelihood ratio tests cannot be used for determining the right number of profiles in person-centered models. The reason is that a model with one profile less can be expressed as the model with one profile more but the parameters of one profile fixed to 0 . In such cases, the difference in likelihoods of two models does not follow a known distribution that would allow computing reliable $p$-values (Peugh \& Fan, 2013). For cross-sectional person-centered models, such as latent class and latent profile analysis, 
adaptations of significance tests are available to determine the best fitting solution (Hickendorff et al., 2018). For example, the bootstrap likelihood ratio test (BLRT) can be used to determine whether there is a significant increase in model fit between the more parsimonious $k-1$ class and the $k$ class model (Nylund et al., 2007). For longitudinal data, as it is used in LTA, such significance tests are not available (Gudicha et al., 2016). The reason is that the BLRT becomes computationally infeasible with large data sets and sample sizes (Tolvanen, 2007; but see Gudicha et al., 2016 for a proposed solution). Instead, commonly employed model fit statistics for latent transition analysis are so-called information criteria, a type of relative model fit index. They are called relative because their values can only be interpreted in comparison to the values obtained from other models, for example when comparing a two- and a three-profile LTA model. The model with lower values on the information criteria has better fit to the data, as they incorporate statistical information of model misfit. There are several information criteria that can be used to assess relative model fit in latent transition analysis. Statistical analysis software usually provides a set of several of these indices (e.g., $A I C, B I C$, and sample-size adjusted $B I C$ in Mplus). Different information criteria draw mostly on the same statistical information when evaluating a latent profile solution: The fit of the model to the data (the likelihood), model complexity (usually the number of parameters), and for some information criteria sample size. Whereas they are based largely on the same statistical information, the indices differ in the ways of combining this information. For example, the commonly used Bayesian Information Criterion (BIC) penalizes model complexity more strongly than the Akaike Information Criterion (AIC) and others (Vandekerckhove et al., 2015). In general, all relative fit indices add penalty to the model likelihood for that depends on the number of model parameters, and sometimes sample size, to find a balance between model complexity anda fit. This raises the question how much 
penalty is the right amount for LTA in educational contexts, and which fit index adds just this right amount of penalty to the likelihood. In the present study, we conduct simulations to examine the adequacy of five of these fit indices for deciding on the number of profiles in latent transition analysis. To help readers understand the commonalities and differences between different relative fit indices, we provide a conceptual overview of the indices included within this study.

\section{Background Information on Model Fit Indices for LTA}

In our study, we compare the rather well-known fit indices $A I C, B I C$, and $a B I C$ (samplesize adjusted $B I C$ ), and, in addition, two further alternatives, the $A I C 3$ and the $C A I C$ (consistent $A I C)$.

The $A I C$ is computed as

$$
A I C=-2 \mathrm{LL}+2 p
$$

where LL is the natural logarithm of the likelihood (the fit of the model to the data) and $p$ is the number of model parameters, reflecting model complexity. The model parameters that have to be estimated in a latent transition analysis (for the model equation, see e.g. Edelsbrunner, 2017 , p. 24; for a description of the model, see supplementary materials S3) are the mean values and variances of each latent profile for each of the indicator variables, the proportions of learners within each of the latent profiles (the last remaining one of which however is not estimated because it just adds up to the full proportion of 100\%), and transition probabilities between all the profiles between all of the measurement points (of which as well the last one always adds up to $100 \%$ ). More complex models generally lead to better overall fit to the data, or a lower $-2 \log$ likelihood. With increasing model complexity, the likelihood of overfitting increases, that is, it becomes more likely to estimate model parameters (in the present cases, profiles and transitions 
between these over time ) that mostly catch nuisance or variance caused by sampling error (Vandekerckhove, Matzke, \& Wagenmakers, 2015). The AIC counters overfitting by putting a penalty of two points on top of the -2LL for each model parameter. This penalty term was meant to select the model that has would be expected to have the lowest error in predicting future data taken from the same population as the sample data (Dziak et al., 2019). This penalty on model complexity is still relatively weak and might be too weak particularly in small samples (Dziak et al., 2019), leading the $A I C$ to prefer rather complex models. This can be seen in Figure 1, where the penalty that different fit indices put on each model parameter is presented.

\section{Figure 1}

Penalty per Model Parameter that the Different Fit Indices Pose on Models, Depending on Sample Size

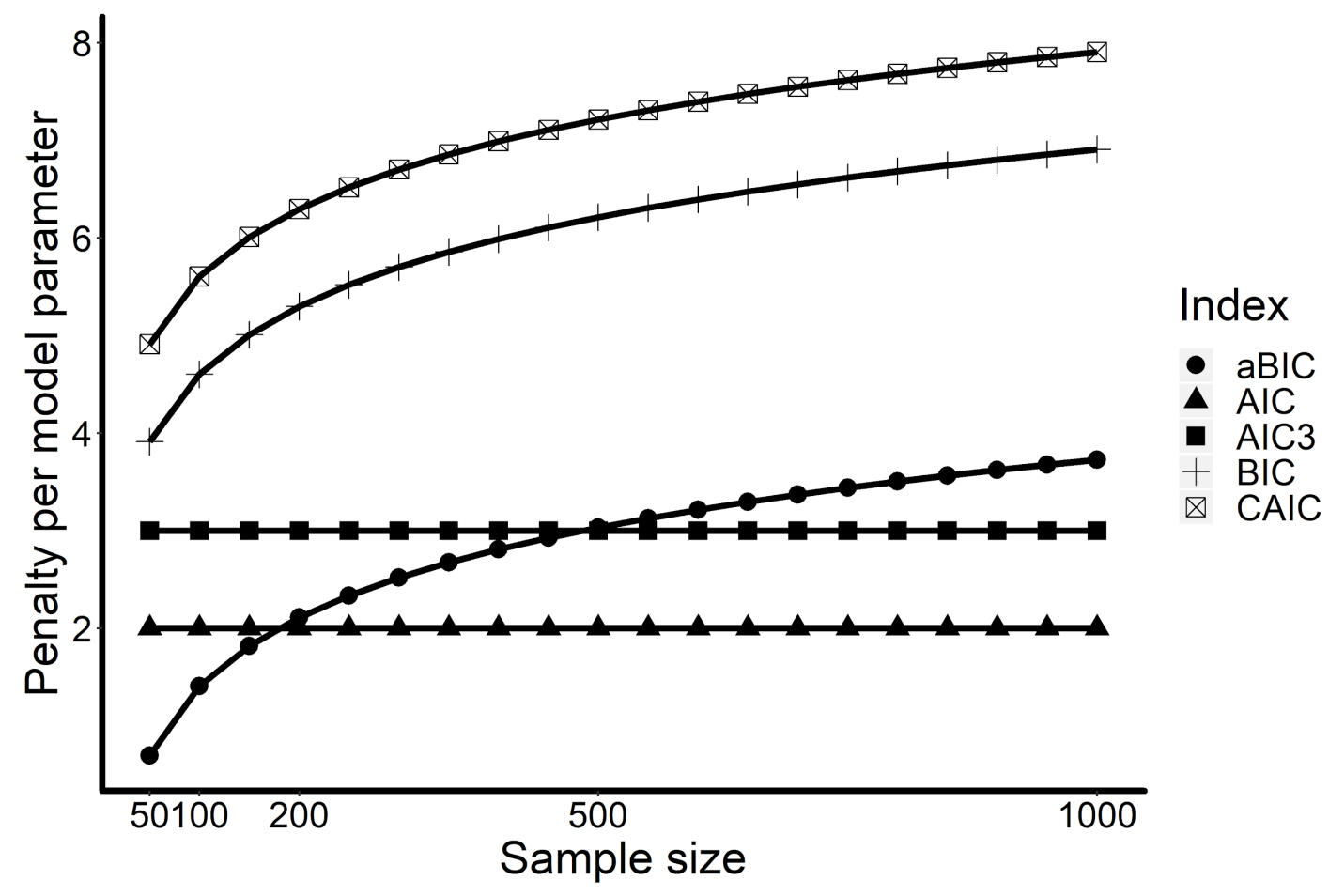

Another popular fit index, the $B I C$, is computed as

$$
B I C=-2 \mathrm{LL}+\log (n) p
$$


This index puts a penalty of $\log ($ sample size $)$ times the number of parameters on top of the -2log-likelihood. This penalty term makes the BIC stricter in terms of model complexity (see Figure 1). The reason is that this index is not meant to provide the best predictive accuracy, which might be better obtained with a weaker penalty, but it is meant to point to the true model of the data-generating process (for a more detailed description, see Aho et al., 2014). Already in small samples of up to $N=200$, this index puts about 4 to 5 points of penalty on each parameter. Consequently, the $B I C$ tends to favor less complex models than the $A I C$, for example, models with a lower number of profiles in LTA.

A third popular fit index, the sample-size adjusted BIC, is computed as

$$
a B I C=-2 \mathrm{LL}+\log ((n-2) / 24) p
$$

The dependence on sample size here is such that in smaller models, the $a B I C$ puts less penalty on each model parameter than the $A I C$, whereas for sample sizes of about $N=200$ and above, it adds more penalty for each parameter than the $A I C$ (Figure 1). Although the adaptation of the penalty term for this index has been developed for time series models, it has been found to work well for example in latent class models (Dziak et al., 2019).

Another alternative is the $A I C 3$ (Fonseca, 2018), which functions similarly to the AIC but just adds three instead of two points of penalty for each model parameter:

$$
A I C 3=-2 \mathrm{LL}+3 p
$$

Whereas the increase in penalty of one point in comparison to the AIC is arbitrary from a theoretical view, the AIC3 has been found to work well in some studies (Dziak et al., 2019).

Finally, the consistent AIC (Vonesh \& Chinchilli, 1997) puts the highest penalty on each model parameter (Figure 1), exactly one point more for each parameter than the BIC:

$$
C A I C=-2 \mathrm{LL}+[\log (n)+1] p
$$


The stronger penalty term in comparison to the $B I C$ has no firm statistical basis (Dziak et al., 2019) but empirically it might work well for some models.

The differences in how these fit indices balance fit, sample size, and model complexity indicate that when comparing the fits of models that differ in the number of extracted profiles/clusters, different model fit indices may indicate different model choices. This can be seen in examples from the applied literature in which the different fit indices disagreed regaring the best solution. For example, Flaig et al. (2018) used latent transition analysis to model university students' development of knowledge about human memory across four time points. They estimated models with increasing numbers of profiles based on three indicator variables that captured students' affirmative answers that were in accordance with a common misconception, a partially correct conception, and a scientifically correct concept of human memory, respectively. The authors estimated models with up to five profiles, resulting in the fit indices shown in Table 1 . The $A I C$ and adjusted $B I C$ indicated that the five profile model had the best fit to the data, whereas the BIC and CAIC favored the three-profile solution. The $A I C 3$ yielded the same lowest value with three and four profiles. This raised the question which fit index should be preferred in this data situation: Should we rely on the more stringent $B I C$ and $C A I C$ regarding model complexity and decide to model three profiles, or should we model five profiles based on the less stringent $A I C$ and $a B I C$ indices? This question is central to the present study: As different fit indices put different amounts of penalty on model complexity, researchers require guidance regarding at which sample sizes (and further factors that may affect precision of fit indices), how much penalty is right for finding the correct number of profiles, and which fit index adds just this right amount of penalty to the respective model log-likelihood. In the study by Flaig et al. (2018), the authors decided to model four profiles, as the additional profile that 
appeared in the four-profile solution compared to the model with three profiles was of theoretical interest. The authors referred to the $B I C$ as a reason not to include the fifth profile.

\section{Table 1}

Fit Indices Obtained in the Study by Flaig et al. (2018)

\begin{tabular}{cccccccc}
\hline $\begin{array}{c}\text { Number of } \\
\text { profiles }\end{array}$ & $\begin{array}{c}\text { Number of } \\
\text { parameters }\end{array}$ & $\begin{array}{c}\text { Log- } \\
\text { likelihood }\end{array}$ & AIC & BIC & aBIC & AIC3 & CAIC \\
\hline 1 & 24 & -1513 & 3074 & 3144 & 3067 & 3098 & 3168 \\
2 & 25 & -1350 & 2750 & 2823 & 2743 & 2775 & 2848 \\
3 & 41 & -1278 & 2638 & 2758 & 2627 & 2679 & 2799 \\
4 & 63 & -1245 & 2616 & 2800 & 2599 & 2679 & 2863 \\
5 & 91 & -1212 & 2606 & 2872 & 2581 & 2697 & 2963
\end{tabular}

Note. The sample size was $N=137$. Fit indices computed based on number of parameters and log-likelihood values reported by authors.

We will come back to this example to see whether the results that we obtain from the present study support the authors' decision, or would have suggested a different solution.

Situations in which different fit indices disagree are rather the rule than the exception when using latent transition analysis (e.g., Schneier \& Hardy, 2013; Edelsbrunner et al., 2018; Flaig et al., 2018). Particularly in small samples $(N s<500)$, under conditions of imperfect measurement (indicator variables with measurement error), study dropout, or model misspecification, fit indices may lack accuracy in determining the correct number of classes or profiles (Peugh \& Fan, 2013). Researchers need to rely on guidelines regarding which model fit indices will most likely reflect the best model choice for an analysis at hand.

\section{LTA in Educational Research: An Applied Example}


For the present study, we construct another example in which we describe hypothetical learner profiles and learners' transitions between these profiles in the course of learning. We base this example on typical design characteristics and results from previous studies that have used LTA, or related cross-sectional models, in educational research (e.g., Flaig et al., 2018; Fryer, 2017; McMullen et al., 2015; Schneider \& Hardy, 2013; Straatemeier et al., 2008; van der Maas \& Straatemeier, 2008). The online supplementary materials provide an extended description of the characteristics of these prior studies and how they influenced the current scenario. This example scenario serves two purposes: (1) It illustrates the questions researchers are faced with when conducting LTA, and (2) it describes the central scenario for data simulations of the current simulation study.

Let us assume that we have collected data in a longitudinal study with a group of learners at three measurement points. The three measurement points represent a pretest before an intervention, a posttest after the intervention, and a follow up-test later after the intervention. The learners answered the same knowledge test at each measurement point. Based on learners' answers, the researchers calculated scores on each of three continuous indicator variables at each individual measurement point. The three indicator variables represent indicators of an idealized developmental progression from preconceptions (Indicator 1) over intermediate concepts (Indicator 2) to scientifically correct concepts (Indicator 3). In this scenario, Indicator 1 represents a measure of early, scientifically incorrect knowledge. Students who are in an early stage of learning in science will show above-average scores on this indicator, for example, a misconception of rational numbers or density (see for example, McMullen et al., 2015; Hardy et al., 2006). Indicator 2 represents a measure of intermediate knowledge. This could be an intermediate concept that is not in full accordance with scientific facts, for example, a physical 
concept of size and matter that does not yet fully integrate these two aspects of density

(McMullen et al., 2015; Hardy et al., 2006; Schneider \& Hardy, 2013). Indicator 3 represents advanced knowledge. For example, this would represent a fully scientifically correct concept of rational numbers or density.

In the population, learners differ on these three indicator variables according to four underlying profiles. This is in line with prior studies, which typically identified two to five profiles (Edelsbrunner et al., 2018; Fryer, 217; McMullen et al., 2015; Schneider \& Hardy, 2013). In our example, each profile reflects a certain quality of knowledge (i.e., from naïve to expert understanding ) and is characterized by its specific configuration of misconceptions (Indicator 1), prescientific concepts (Indicator 2), and scientific concepts (Indicator 3). These profiles are depicted in Figure 2; note that the indicators are centered around an arbitrary mean of 50.

\section{Figure 2}

Four Latent Profiles, Defined by Patterns of Mean Values Across Three Indicator Variables

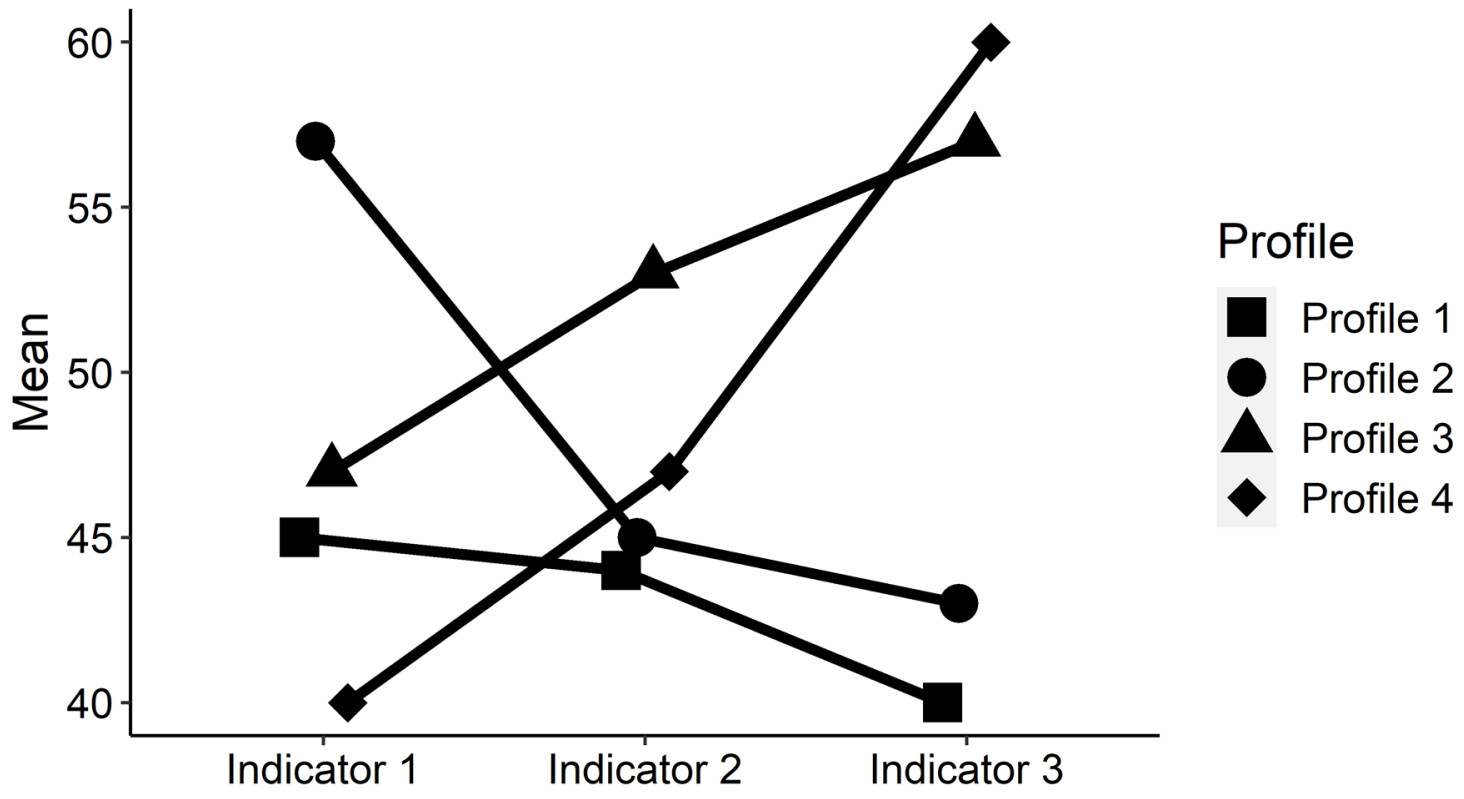




\section{Latent Profiles}

Profile 1 describes learners who show low scores on all three indicator variables, misconceptions, prescientific concepts, and scientific concepts. This represents learners who have not yet developed clear concepts, beliefs, or knowledge about a topic. Consequently, neither a high level of misconceptions nor high levels of more advanced learning progress are visible in these learners. Such profiles have been found in prior studies across various topics (Flaig et al., 2018; Edelsbrunner et al., 2018; Schneider \& Hardy, 2013). Profile 2 describes students in an early stage of learning with naïve understanding, who show high levels of misconceptions (Indicator 1). Such profiles are commonly found in science education (Straatemeier et al., 2008; van der Maas \& Straatemeier, 2008). Profile 3 describes learners who are in an intermediary stage of learning, in which they show rather high levels of prescientific concepts (Indicator 2) but also high levels on scientific concepts (Indicator 3; Edelsbrunner et al., 2015; Schneider \& Hardy, 2013). Profile 4 represents a group of advanced students who have reached an (almost) expert understanding (Flaig et al., 2018).

\section{Latent Profile Transitions}

At each of the three time points, students can belong to one of these four profiles. Educational researchers are usually interested in learners' transitions between profiles over time. Transitions between profiles can for example represent learning (from pre- to posttest) or forgetting (from posttest to follow up), depending on the nature of the profiles. Staying in the same profile can be an indicator of the absence of learning processes (from pre- to posttest), and transitioning into an initial profile may indicate a forgetting process (from posttest to follow up). As in our example, we assumed that there is an intervention between the first and the second measurement point, learners will most likely transition to another profile between T1 and T2. 
Under the assumption that the intervention is fairly effective, most learners will transition from less advanced profiles to more advanced profiles (e.g., from Profile 1 to Profile 3 or 4). The resulting transition probabilities from $\mathrm{T} 1$ to $\mathrm{T} 2$ are depicted in Figure 3. Also, as there is no further intervention, we expect that these changes will remain relatively stable between T2 and the follow-up measure at T3. Consequently, we will find high probabilities for learners to stay in the same profile between T2 and T3, with lower probabilities for transitioning back to lowquality profiles (reflecting forgetting or regress), and also lower probabilities for advancing to higher quality profiles (delayed learning).

\section{Figure 3}

Simulated Transitions Between the Four Profiles Across the Three Measurement Points

T1 Intervention T2 Follow up T3

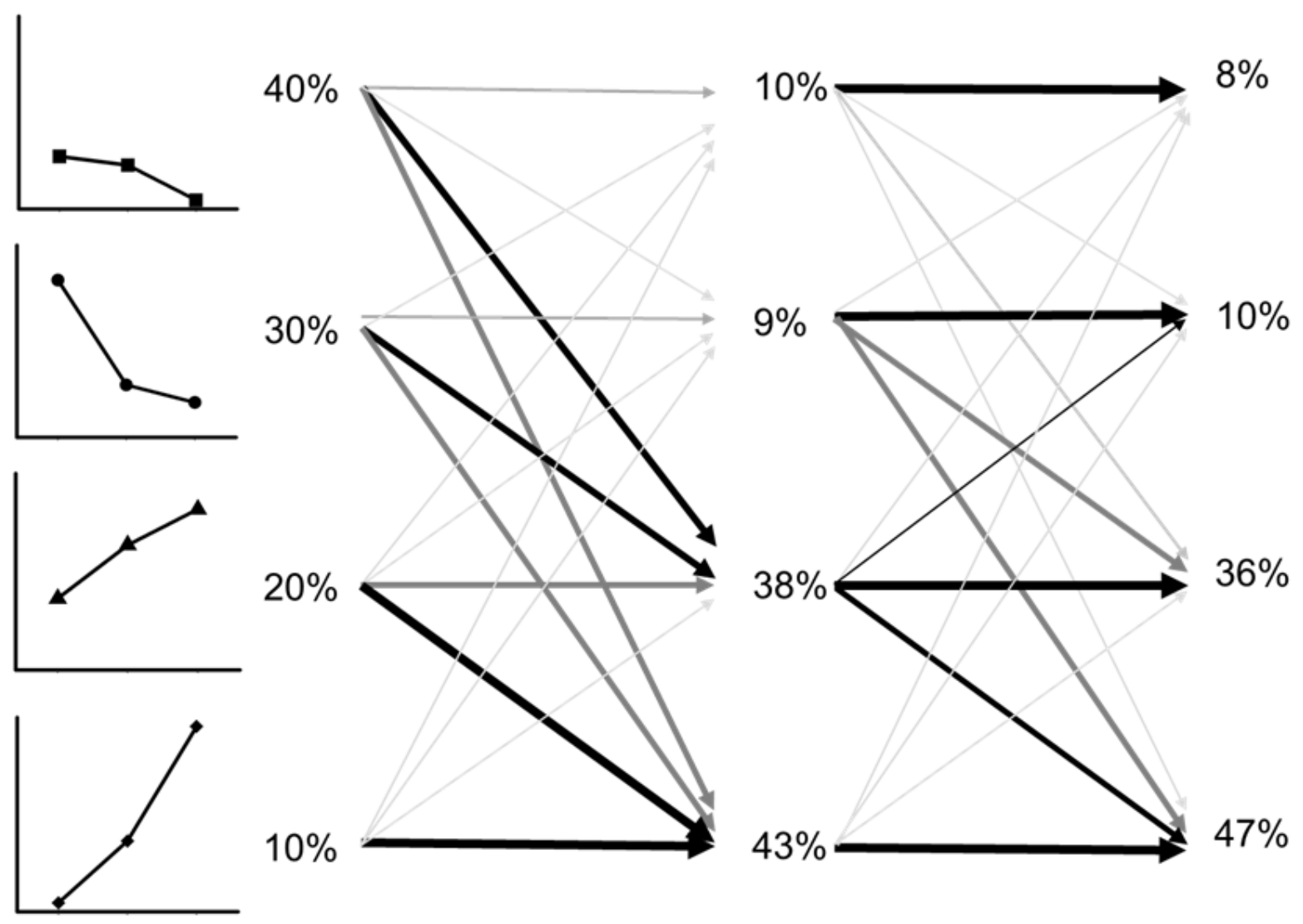


Note. Thicker lines indicate higher probabilities of transitioning between two profiles between two time points. Thinnest lines indicate 5\% probability, thickest line 95\%. Percentages at each time point indicate the resulting percentage of learners showing each profile at the respective measurement point.

\section{Conducting the Analysis}

As researchers conducting this example study, we would be unaware of the true nature of the profiles in the population (as described in the previous paragraphs and the example by Flaig et al., 2018). The question is thus, how we should decide on the number of profiles to be extracted in the analysis? From a mathematical perspective, many solutions are possible. Assuming for simplicity that learners show either low, average, or high scores on the Indicators $1-3$, then there are $3^{3}=27$ possible profile configurations at each measurement point. To analyze the underlying knowledge profiles and transition patterns throughout the three measurement points, the researcher would set up an LTA model. To allow a straightforward interpretation of the transition patterns, the researchers would constrain the indicator means and variances of each profile to be equal across the three time points. This represents an assumption of measurement invariance, which can also be tested (Collins \& Lanza, 2010). Thus, the interpretation of the profiles would not change throughout the study, that is, a misconceptions profile would show the same configuration of the Indicators 1-3 at T1, T2, and T3.

To determine the number of profiles in our example, the researchers would conduct LTA and compare the fit of models with different numbers of profiles, for example, two, three, four, and five profiles. Probably, they would do some exploratory analyses with single time points first before they set up the longitudinal model to decide on the number of profiles in the final model. 
As there is no significance test available for LTA models, the researchers would rely on the information criteria when deciding on the number of profiles. When plotting the fit statistics of the different models, they might see an increase in fit when adding more profiles, for example an increase in fit between the five-profile and the four-profile model. However, different fit indices may favor different model choices. For example, the $B I C$ might indicate that the three-profile solution has the best fit to the data, whereas the $a B I C$ might point towards four-profile solution, and the $A I C$ towards five-profiles. For this reason, simulation studies are needed that help researchers decide which of the fit indices (or a combination thereof) works best for reliably finding the correct number of profiles.

\section{Performance of Fit Indices: Evidence from Simulation Studies}

Simulation studies can be a useful means to guide researchers' reliance on specific fit indices in their modeling decisions. They provide information on how fit indices perform under various modeling conditions (e.g., sample size, amount of missing data, class/profile separation, number of measurement occasions, number of observed variables). One frequently cited publication is a Monte Carlo simulation study conducted by Nylund et al. (2007). From their analyses, the authors derived recommendations concerning the use of likelihood-based tests and information criteria in different mixture models, encompassing latent class analysis, factor mixture models, and growth mixture models. The $B L R T$ emerged as the best performing index for model selection, followed by the $B I C$ and adjusted $B I C$ across the different scenarios (varying sample sizes and numbers of items) under consideration. Perhaps the consistency of these results in various modeling scenarios is a reason why the findings of the study are commonly cited in the selection process in mixture modeling (see Edelsbrunner et al., 2015; Flaig et al., 2018; Kainulainen et al. 2016; McMullen et al., 2015; Mellard et al., 2016; Schneider 
\& Hardy, 2013; Swanson, 2012). Moreover, the findings of some other simulation studies are largely consistent with Nylund et al. (2007) in cross-sectional (Peugh \& Fan, 2013; Tein et al., 2013 ) and longitudinal designs (Chen et al., 2017).

However, the study by Nylund et al. (2007) did not actually examine the performance of fit indices for LTA. Their study only encompassed the other mentioned kinds of mixture models. We are not aware of any simulation studies that have investigated the performance of fit statistics for identifying the correct number of classes/profiles specifically in LTA. Findings from other simulations with latent Markov models (generalizations of LTA) suggest that characteristics of the longitudinal design, such as the sample size, the number of observed variables, and the number of measurement occasions, as well as characteristics of the population model (i.e., the number of latent variables, initial proportions of the latent variables, stability of latent variable membership) are critical determinants of power when testing hypotheses in these models (Gudicha, Schmittmann, \& Vermunt, 2016). Furthermore, some studies noted that the BIC should be used with caution under certain conditions, for example because of a tendency of underfitting or generally unsatisfactory performances of all fit statistics examined (Bacci et al., 2014; Lin \& Dayton, 1997; Morgan, 2015; Peugh \& Fan, 2013; Tein et al., 2013). Thus, it is a matter of scientific inquiry to investigate whether findings from prior simulations conducted in cross-sectional scenarios or using other longitudinal models can be extended to latent transition analysis, particularly within the context of educational studies.

\section{The Current Study}

The current study tackles this lack of knowledge concerning the central aspect of identifying the correct number of latent classes/profiles in latent transition analysis. In this study, we simulate data from a typical scenario in educational research, specifically the hypothetical 
scenario described before. In this scenario, we investigate the research question of how different fit indices perform in identifying the correct number of classes/profiles under varying conditions of sample size, participant dropout, and in the presence of more or less distinctive profiles. In addition, we will provide a robustness check to examine whether our obtained results might generalize to different scenarios and to bring up related hypotheses for future research.

\section{Method}

\section{Simulation scenario}

We simulated data from the scenario presented in the applied example above. This scenario can be considered typical for educational research, as it resembles the average numbers of time points, indicator variables, and profiles, as well as typical profile patterns found in educational research (Compton et al., 2008; Edelsbrunner et al., 2015; Edelsbrunner et al., 2018; Flaig et al., 2018; Fryer, 2017; Gillet et al., 2017; McMullen et al., 2015; Kainulainen et al., 2016). In our scenario, we assumed measurement invariance. In LTA, this means that the number of profiles, as well as the mean and variance parameters characterizing the profiles remain the same across all measurement points (Collins \& Lanza, 2010). We simulated four profiles and transitions of individuals between these profiles across three measurement points as presented in Figures 2 and 3.

\section{Simulation conditions}

Whereas the described scenario was the same across all simulation runs, we varied three factors that represented our experimental simulation conditions: Sample size, entropy (profile separation), and participant dropout. An overview of the simulation conditions is presented in Table 2 . 


\section{Table 2}

Overview of Simulated Scenarios and Study Conditions Within Those Scenarios

\begin{tabular}{lccc}
\hline \multicolumn{1}{c}{ Standard scenario } & Sample sizes & Entropy & Attrition \\
\hline $\begin{array}{l}\text { indicator variables, } \\
4 \text { latent profiles }\end{array}$ & $N=50,100,200,500$, & & $\begin{array}{c}\text { No attrition/ } \\
10 \% \text { attrition per }\end{array}$ \\
\hline Robustness check & 1000 & $E=.73, .82, .87$ & wave \\
\multicolumn{1}{c}{ scenarios } & Poor conditions & Moderate conditions & Good conditions \\
\hline $\begin{array}{l}1,2 \text {, or 4 time points, } \\
4 \text { indicator variables, }\end{array}$ & & & \\
alternative profiles, & $N=200, E=.73$, & $N=500, E=.82$, & $N=1000, E=.87$, \\
3 or 5 latent profiles & $10 \%$ attrition/wave & $10 \%$ attrition/wave & no attrition \\
\hline
\end{tabular}

As a first experimental variation, we simulated data with five different sample sizes $(N=$ $50,100,200,500,1000)$. These sample sizes covered the typical sizes found in the published literature applying latent transition analysis in education. We also included a condition with a very small sample size of only $N=50$ because we sometimes encounter researchers who are interested in applying methods such as latent transition analysis to such small datasets and who would like to know whether this might yield informative results. We caution however that with an $N$ of only 50, the number of estimated parameters in a latent transition analysis can easily be larger than the number of observations (i.e., the sample size). In this case, many model estimators will show identification or convergence problems.

The second experimental variation in the simulations was entropy, or profile separation (Collins \& Lanza, 2010). The strength of the differences between profiles is not just determined by their mean value patterns, but also by the variances of learners within the different profiles. If variances within the profiles are high, this implies that their overall distributions still overlap 
strongly despite the differences in mean values. If variances within the profiles are low, there is less overlap in their overall distributions, and the mean value patterns are more distinct. In the former case, when the differences in mean values are rather small compared to their variances, this results in low profile separation. The average separation, or distinctiveness of profiles in an LTA model is summarized within an index called entropy. If entropy is high (close to 1), individuals belonging to different profiles can be clearly separated based on their patterns across the indicator variables. Entropy is essentially a weighted average of individuals' posterior probabilities to belong to a certain profile given their observed patterns of values across the indicator variables (Collins \& Lanza, 2010, p. 75). The formula of the entropy-index is provided in the supplementary materials S1. If entropy is low (further away from 1, closer to 0), individuals belonging to different profiles do not so clearly show different patterns across the indicator variables, because the variances of the indicator variables overlap more strongly. We varied the variances within the four profiles, resulting in entropy values of .73 (low entropy), .82 (medium entropy), or .87 (high entropy). These values encompass most of the range of entropy estimates from prior studies applying LTA in education (see supplementary materials S2). We did not follow any formal rules in setting the variances and fixing entropy. Instead, to make the simulated scenario as realistic as possible, variances differed between the profiles, with those variances belonging to profiles with more extreme means fixed to be smaller (reflective for example of floor and ceiling effects on indicator variables) than those closer to the grand mean. We did not impose parameter constraints apart from measurement invariance over time.

The last experimental variation in the simulations was sample attrition. In educational research, the dropout of participants over time often cannot be fully avoided. In the published literature applying LTA in educational research, participant dropout varied between $0 \%$ and 
about $30 \%$. We included one simulation condition in which there was no attrition, and a second one with attrition. We modeled random attrition of $10 \%$ at the second and another $10 \%$ at the third measurement point, summing up to $20 \%$ of missing data after the third measurement point (this was the typical amount of missing data in prior studies, see supplementary materials S2).

In addition to these simulation conditions, we varied further factors in a less comprehensive manner to provide a first look into the robustness of our findings and bring up hypotheses for future research. This included the number of indicator variables in the analysis, the number of measurement points, as well as the number and nature of profiles in the population (e.g., non-linear or linear profiles, such that patterns of profile means either overlap or do not overlap across indicator variables; Hickendorff et al., 2018).

\section{Simulation Strategy}

We simulated (i.e., randomly generated applying monte carlo-simulation) data sets for each of the 30 (five sample sizes, three entropy levels, attrition or no attrition) conditions. Subsequently, we analyzed each dataset with latent transition analyses with varying numbers of profiles. In this way, we examined how frequently different fit indices point towards the correct solution with four profiles under each condition. We simulated and analyzed between 300 and 600 data sets for each combination sample size, entropy, and attrition. We simulated and analyzed all data using Mplus 8.5 (Muthén \& Muthén, 2021) via the MplusAutomation (Hallquist \& Wiley, 2018) and tidyverste (Wickham et al., 2019) packages in the R software environment (R Core Team, 2021). The number of simulated datasets was restricted by the running time of the simulations in Mplus. Although this software package is known for its fast estimation procedures for models of the kind in focus here, the estimation of a single model lasted between one minute and 30 minutes using multithreading on a 16-core $2.70 \mathrm{GHz}$ Intel 
Xeon Gold 6150 Windows desktop computer with 128 RAM. All syntaxes and results-objects for $\mathrm{R}$ can be found under

https://osf.io/w9uqy/?view_only=6c3cbdb1284346db98c7396346d06de1. Concerning the technical implementation of the simulations and analyses, we used maximum likelihood estimation with an expectation-maximization algorithm, with 400 random starts of which the most promising 100 were continued for estimation until the relative loglikelihood-change between two iterations was less than $10^{\wedge}-4$ and the absolute change less than $10^{\wedge}-4$. Further technical details are provided in the supplementary materials.

On each dataset, we estimated latent transition analyses with three to five latent profiles at each time point. For each estimated model, we computed the five fit indices discussed above (AIC, BIC, aBIC, AIC3, CAIC) and examined for each index whether it favored the model with three (underfitting), four (correct model choice), or five (overfitting) profiles.

\section{Robustness check}

As a robustness check, we then selected three scenarios from our original set of factors corresponding to poor, moderate, and ideal conditions, and then verified our findings for these scenarios while changing five different aspects of the data generating process. First, we varied the number of measurement points. With only one measurement point, an LTA reduces to a latent profile analysis; latent student profiles are estimated but no transition probabilities over time, because the data are cross-sectional. To examine the robustness of our results towards this cross-sectional design, we analyzed just the first measurement point of the simulated data from the standard scenario with a typical latent profile analysis model. To further examine the robustness within a design with two measurement points, we also analyzed only the first and second measurement point of the simulated data from the standard scenario applying latent 
transition analysis. Finally, to examine robustness for a study design with four measurement points, we added a fourth measurement point to the standard scenario. We then simulated transition probabilities that resembled a longitudinal study design in which there is no intervention but more steady change between measurement points. The second characteristic that we varied for the robustness check was the number of indicator variables. We set up a scenario with a fourth indicator variable that represents another intermediate step in an educational progression, for example in building up knowledge or a skill. Details of the profiles that we set up, which resemble those for the standard scenario but with this additional intermediate indicator variable, are described in supplementary materials section S2. The third characteristic that we varied for the robustness check was the number of simulated profiles. To this end, we removed one of the profiles (profile 3) but the other three profiles and their transition probabilities across the three measurement points remained the same as in the standard scenario. We also simulated a scenario with five profiles; in this scenario, the fifth profile describes learners with low values on all indicator variables. As a fourth and final characteristic, we varied the nature of the simulated profiles. To this end, we set up a scenario within which the four profiles represent motivational/affective states in which there exists less overlap than in the profiles of the standard scenario. In this way, we covered a different kind of educational research scenario in which person-centered approaches such as LTA have also been used recently (e.g., Gillet et al., 2017) and at the same time we covered profiles with less overlap/non-linear patterns of mean values across the indicator variables. An overview of the overall seven scenarios of the robustness check is provided in Table 1.

For the robustness check, we simulated and analyzed 300 datasets each for three benchmark conditions from among the 30 conditions of the standard scenario: Poor conditions, 
with a sample size of $N=200$, low entropy of .73 , and missing data with $10 \%$ dropout per wave; moderate conditions, with a sample size of $N=500$, medium entropy of .82 , and missing data with $10 \%$ dropout per wave; and good conditions, with $N=1000$, high entropy of .87 , and no missing data.

\section{Results}

We report the results in three parts. First, we present the accuracy of the five fit indices under the different conditions. Accuracy is the proportion of simulated datasets for which a fit index points towards the correct model (i.e., with four profiles) under the different conditions. We summarize the accuracies of the fit indices in increasing magnitude of sample sizes. In the second part, we examine in detail why some fit indices perform better than others. To this end, we summarize the frequencies of overfitting (selecting the model with five profiles) and underfitting (selecting the model with three profiles) for the five fit indices. In the third part, we present the results for the robustness check.

\section{Accuracy of the Five Fit Indices Across Conditions}

As the simulation outcomes showed, at a sample size of $N=50$, the AIC is the only fit index that could correctly identify the model with four profiles in more than $50 \%$ of cases (see Figure 4). At this extremely small sample size, all other fit indices constantly show extremely low accuracies below $15 \%$. 


\section{Figure 4}

Accuracy (Proportion of Correctly Identified Models) for the Fit Indices Across All Thirty

Conditions of Sample Size, Entropy, and Attrition
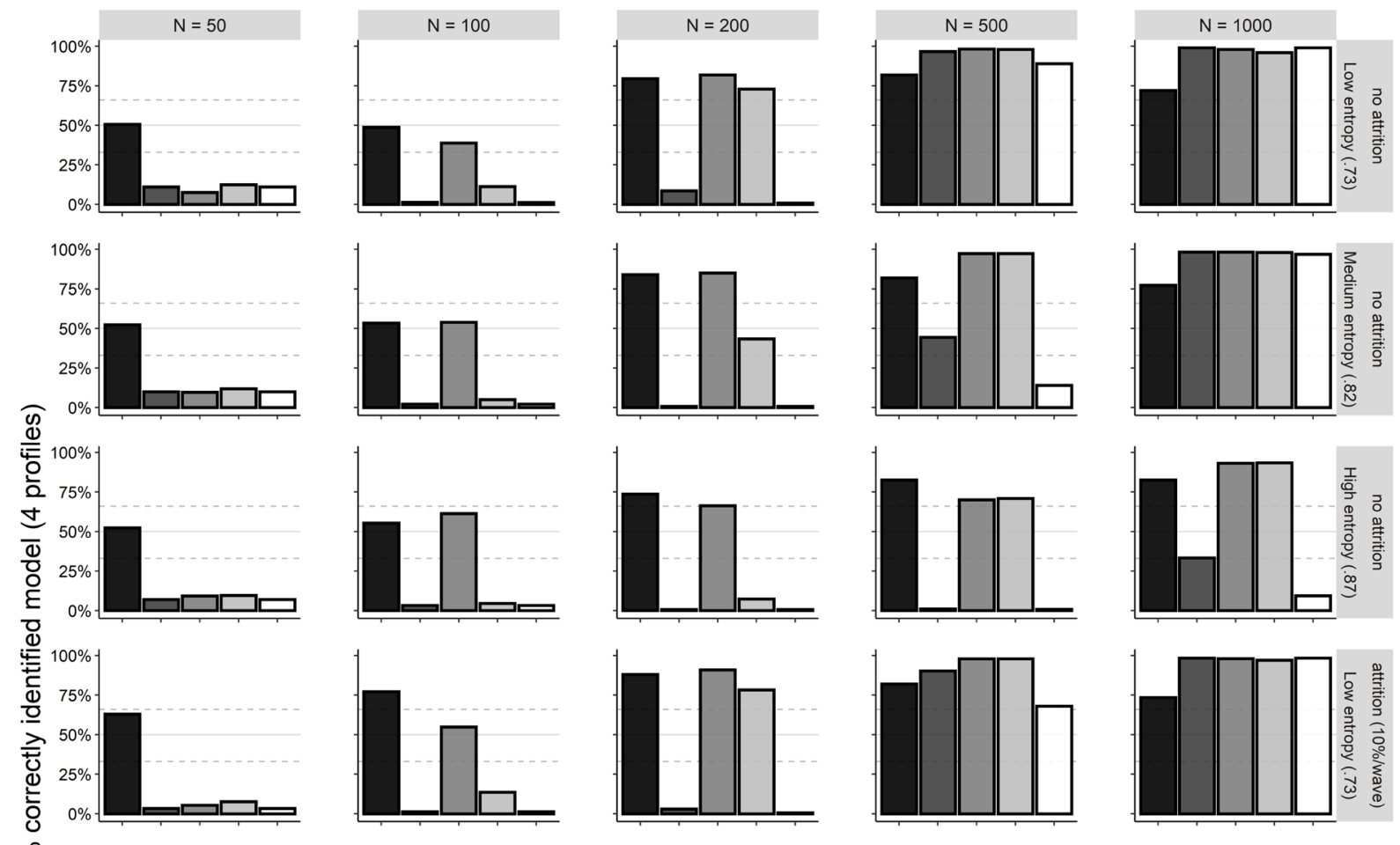

Fit index

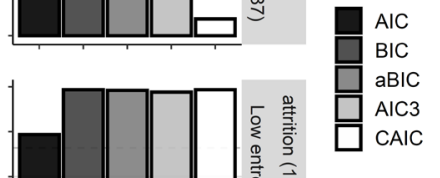

$$
\text { ๖ } 100 \%
$$
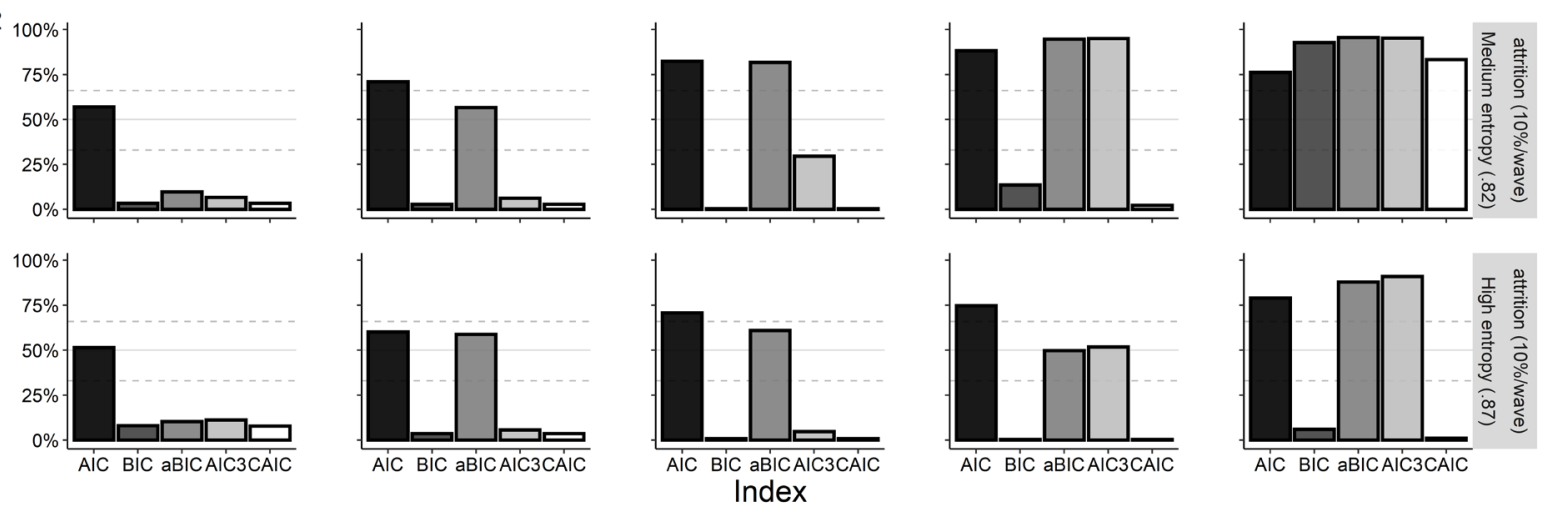

Note. Full horizontal line indicates 50\% accuracy, dashed lines 33\% and 66\%. 
With $N=100$, the accuracy of the $A I C$ increases to about $75 \%$ across conditions, and the aBIC now is also constantly above $50 \%$, apart from the condition with low entropy and no attrition. The accuracy of the remaining fit indices remains very low.

With $N=200$, the $A I C$ and the $a B I C$ are constantly above $75 \%$ of correctly identified models, apart from the high entropy condition under which the performance of both appears to decrease slightly. At this sample size, also the $A I C 3$ works better, however its performance depends as well substantially on entropy. At the lowest level of entropy (.73), the AIC3 achieves accuracies around $75 \%$, whereas its accuracy drops considerably below $75 \%$ in conditions of medium and high entropy. Furthermore, the $A I C$ and the $a B I C$ benefit from attrition in smaller samples of up to $N=200$. This is visible in higher accuracy of these two fit indices in the lower three rows compared to the upper three rows of Figure 4 at sample sizes $N=50$ to $N=200$. At larger sample sizes, however, the most strict model parameters $B I C, A I C 3$, and $C A I C$ appear to suffer moderately from attrition.

At $N=500$, the $A I C, a B I C$, and $A I C 3$ all demonstrate rather high accuracies. With low and moderate entropy, the $a B I C$ and the $A I C 3$ outperform the $A I C$, whereas with high entropy, the $A I C$ outperforms the two other indices. At this sample size, the $B I C$ and the $C A I C$ finally show better accuracies. However, both seem to suffer from higher entropy levels. The CAIC only performs well under low entropy, and the BIC under low to medium entropy. In addition, both the $B I C$ and the $C A I C$ perform worse under attrition than without attrition.

At the largest sample size, with $N=1000$, all fit indices generally perform well. Only under high entropy, particularly in combination with attrition, the $B I C$ and the $C A I C$ lack accuracy, similarly to the patterns that they showed for $N=500$. 
Since sample size appeared to be the decisive factor in determining the propensity of the different fit indices to find the right model, in Figure 5 it is shown how the indices behave across different sample sizes, averaged across the other factors.

\section{Figure 5}

Propensity to Indicate the Correct Model or Under-and Overfit for Different Fit Indices Across Sample Sizes

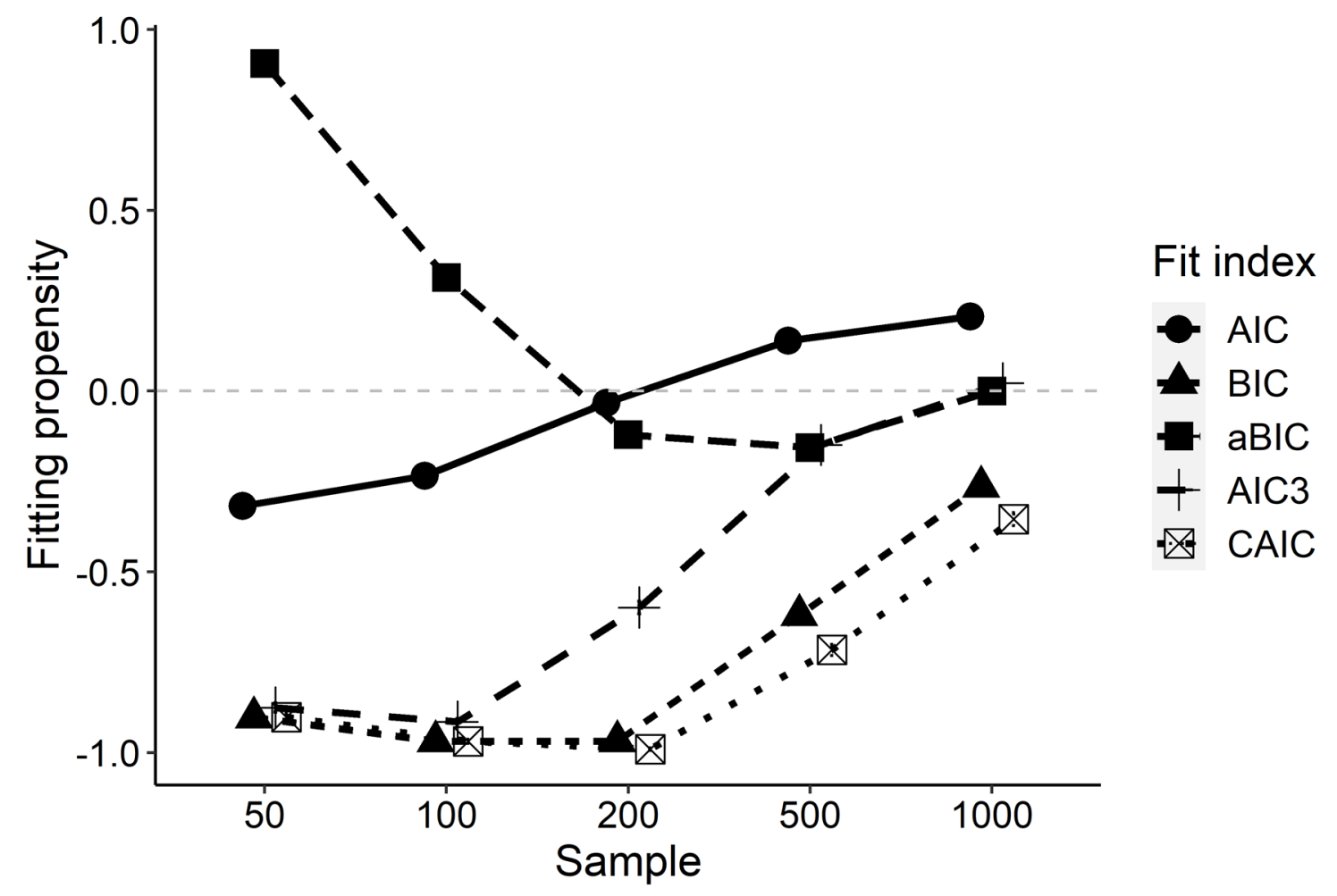

Note. 0 -line indicates perfect calibration of fit index at respective sample size, $>0$ propensity to overfit, $<0$ propensity to underfit.

Marginalizing across entropy and missingness conditions, we indicated for each fit index applied to each simulated data set whether the index indicated the correct model with four profiles (indicated by a 0), underfitted (indicated by a -1), or overfitted (indicated by a 1 ). Averaging these values for each fit index at each sample size, we can see from Figure 5 under what sample sizes the respective fit indices tend to be overly strict (resulting in an average below 
0 ), tend to find the right model (resulting in values just around 0), or tend to overfit (resulting in values greater than 0). As Figure 5 indicates, the AIC tends to slightly underfit but is the most precise index up to $N=200$, where the AIC and the aBIC (whichc overfits up to $N=200$ ) are both perfectly on spot. At $N=500$ and $N=1000$, the AIC starts to engage in overfitting, whereas in accordance with Figure 4, the AIC3 becomes very precise and yields very similar precision as the aBIC.

\section{Over- and Underfitting of the Five Fit Indices Across Conditions}

Figures S2 to S6 in the supplementary materials represent the frequencies of the fit indices regarding underfitting, overfitting, and identification of the correct model across the various conditions in more detail.

AIC. Overall, it is visible that in accordance with Figure 5, the reason for the moderately good, yet limited performance (50 - 70\% of correctly identified models) of the AIC under small sample sizes is underfitting (see Figure S2). This ties back to the penalty of two points that the $A I C$ puts on each model parameter (see Figure $1 \&$ Equation 1). In samples of up to $N=100$, the $A I C$ tends to underestimate the number of profiles. At sample sizes of $N=200$ and $N=500$, this is not the case and $A I C$ exhibits almost optimal performance, whereas with $N=1000$, overfitting emerges.

BIC. As shown in Figure S3, the BIC tends to underfit (bars on grey) in smaller samples (up to $N=200$ ). We observed an interaction between sample size and entropy on the accuracy of the $B I C$ in larger samples of $N=500$ and $N=1000$. Whereas the performance of the $B I C$ was almost perfect in samples with low entropy, underfitting reemerged in samples with medium and high entropy $(N=500)$. Note that the $B I C$ puts strong penalty of about six points on each model parameter in such large samples (Figure 1, Equation 2). When increasing the sample size to $N=$ 
1000 , where the penalty per model parameter increases to about seven points (see Figure $1 \&$ Equation 2), underfitting occurred only in conditions with high entropy.

$\boldsymbol{a B I C}$. The $a B I C$ showed overfitting (bars in black) in very small samples $(N=50$; Figure S4). In samples of $N=100$, where similar to the $A I C$ this $a B I C$ puts about two points of penalty on each model parameter (see Figure 1 \& Equation 3), its accuracy was never far above chance. Similar to the $B I C$, in conditions with high entropy and a sample size of $N=500$, underfitting emerged, but only in two conditions.

AIC3. The pattern of the $A I C 3$ 's accuracy was similar to the $B I C$, showing underfitting in various conditions (Figure S5). In larger samples, the interaction between sample size and entropy was not as strong as for the $B I C$. Note that the $A I C 3$ always puts less penalty on model parameters than the $B I C$, particularly in larger samples (Figure $1 \&$ Equation 4 ). In samples of $N$ $=1000$, the performance of the $A I C 3$ was almost perfect and very reliable across conditions (Figure 8).

CAIC. The pattern of the $C A I C$, which always penalizes each model parameter more strongly than the $B I C$ (Figure 1 \& Equation 5), was similar to the $B I C$ and $A I C 3$ but with stronger underfitting except for a few conditions with large samples $(N=1000$, low to medium entropy; Figure S6).

\section{Robustness Check}

The results regarding the accuracy of the fit indices in the seven robustness checkconditions are provided in Figure 6 (the more detailed results for individual fit indices are provided in supplementary materials section S4). To summarize the results from these conditions, the main conclusions from the standard scenario appear to hold. The $B I C$ and the $C A I C$ underestimate the actual number of profiles, particularly with just one measurement point 
(i.e., a latent profile analysis, with no transitions over time), with only three latent profiles, and more moderately with two measurement points or four indicator variables .

\section{Figure 6}

Accuracy of Fit Indices in the Seven Robustness Check-Scenarios
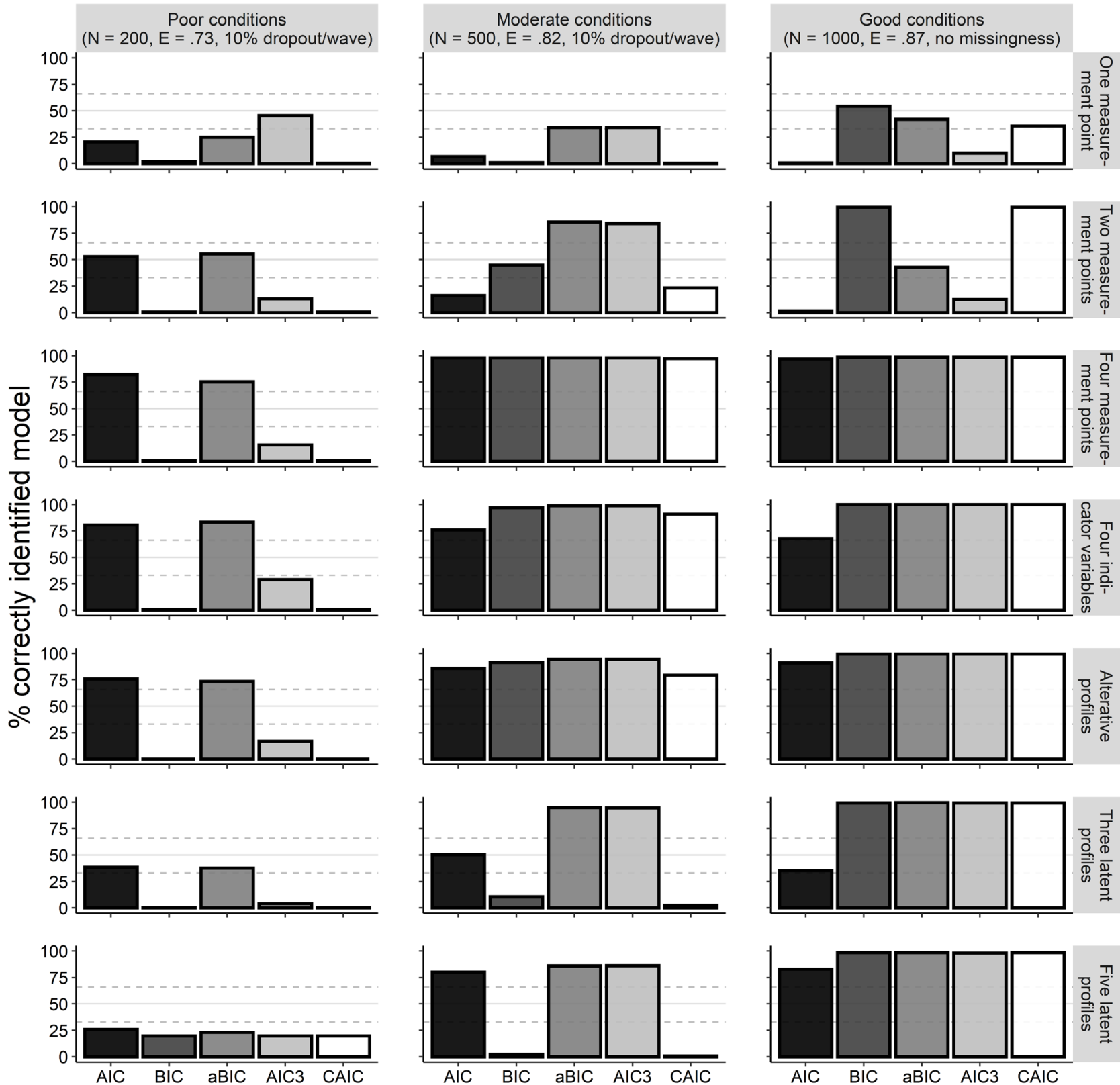

Note. Full horizontal line indicates 50\% accuracy, dashed lines 33\% and 66\%. 
The $A I C$ works well overall but overestimates the number of profiles with only one measurement point, more moderately with two measurement points , and also moderately with three latent profiles. The $a B I C$ works well under all scenarios. However, it tends to overfit with one measurement point. The $A I C 3$ shows a mixed picture with both over- and underfitting with only one or two measurement points, but it works well under the further scenarios apart from poor conditions. Overall, it appears that under scenarios with more linearity in the profiles (with four indicator variables and in the alternative scenario of affective/motivational profiles), as well as with four measurement points (i.e., more repeated observations of the profiles), all fit indices work better than under the standard scenario.

\section{Discussion}

\section{Key Findings}

To our knowledge, this study is the first simulation study on the performance of different fit indices for latent transition analysis applied to educational research. Overall, the results are rather clear and consistent. The $A I C$ and $a B I C$ showed high accuracy in smaller samples, the $A I C 3$ in larger samples. The BIC and CAIC showed substantial underfitting, which decreased only under some conditions with the largest sample sizes and low entropy. We now discuss the more detailed results for each individual fit index and then comment on limitations of this study and implications for educational research.

\section{Performance by Fit Index}

Overall, the accuracy of the fit indices increased with increasing sample size. In very small samples $(N=50)$ the probability of identifying the correct model with four profiles was below $50 \%$ for all fit indices. In small samples $(N=100)$, only the performance of the $A I C$ was more appropriate (50-75\% accuracy) but far from perfect. Over- or underfitting in such small 
samples is more likely than identifying the correct model. In larger samples from $N=200$ on, accuracy generally increases. Nevertheless, not each fit index is reliably precise in identifying the correct model in large samples of $N=500$ and $N=1000$. In such large samples, our results indicate moderate overfitting for the $A I C$, an interaction between sample size and entropy on the performance of the $B I C$ and $A I C 3$, and overall insufficient accuracy of the $C A I C$.

The varying results between the fit indices can be traced back to how they are computed. In our descriptions of the equations of the different fit indices, we pointed out that they differ in the amount of penalty that they put on every model parameter. Our results reflect the different amounts of penalty and how they vary across samples sizes for some of the fit indices. From this view, our results can be discussed based on the question of how much penalty is adequate at what sample size. In smaller samples, the $B I C, A I C 3$, and $C A I C$ showed strong underfitting, the AIC moderate underfitting, and the $a B I C$ overfitting. This can be traced back to the fact that in small samples, the $B I C, A I C 3$, and $C A I C$ put higher penalty on each model parameter than the $A I C$ and the $a B I C$. Apparently, the resulting penalty is too high, causing these fit indices to prefer models with too few profiles in small samples. The $A I C$, which puts less penalty on each model parameter, performed best in smaller samples, whereas the $a B I C$ puts even less and too little penalty on each parameter and therefore resulted in more overfitting. In larger samples, the penalty term of the AIC3 of exactly three points for each model parameter appears to be just right for selecting the correct model. The aBIC has the same amount of penalty at $N=500$, which is reflected in the comparable results for these two indices at that sample size. In contrast, starting from this sample size, the two points of penalty that the $A I C$ puts on each model parameter are too little and this index produces overfitting. 
These findings are generally in line with prior simulation studies pointing towards underfitting for the $B I C$ in related kinds of models (Bacci et al., 2014; Lin \& Dayton, 1997; Morgan, 2015; Peugh \& Fan, 2013; Tein et al., 2013). The consistency of our results with the penalty terms that the different fit indices work with and with prior findings speak for their validity.

\section{Limitations and Future Outlook}

We restricted this simulation study to a design with a single group. This does not exclude our findings from informing experimental research involving multiple groups or treatments. Data from experimental studies can be analyzed with a latent transition analysis assuming a single group of students by estimating LTA models first and then relating the model parameters to a group indicator variable, for example via the three-step approach (Nylund-Gibson et al., 2014). The effects of an intervention could then, for instance, be visible in varying transition probabilities across groups, or in profiles that are reached only by individuals receiving a specific intervention (Grimm et al., 2021).

We did not assume a hierarchical data structure, for example with students nested in classrooms or in schools. Simulation studies have shown that neglecting hierarchical data structure in similar models can lead to biased results. This problem occurs only under specific circumstances (Chen et al., 2017). Future studies should evaluate to what extent our findings generalize to such contexts.

Future research should consider our result that the fit indices with higher penalty terms $B I C, A I C 3$, and $C A I C$ decreased in performance under higher entropy values. Apparently, their high penalty terms come into play when the variances within profiles overlap less strongly (i.e., entropy is higher). These indices then showed underfitting. One reason for this might be that the 
additional variance parameters of each added profile in the model contribute less to good model fit. There is less variance to explain around each profile's estimated mean value. The indices, therefore, punish those parameters too much. This and further explanations for overfitting under high entropy should be evaluated in future research.

In our simulations, we considered a specific scenario, informed by published studies applying latent transition analysis in educational research (Edelsbrunner et al., 2015, 2018; Flaig et al., 2018; Kainulainen et al., 2016, McMullen et al., 2015; Schneider \& Hardy, 2013). For example, we simulated data stemming from three continuous indicator variables at pre-, post-, and follow up, and a rather specific pattern of four profiles that exhibit strong non-linear overlap in their mean values across the indicator variables, heterogeneous variances, and measurement invariance over time. It is not yet known whether our results can be fully generalized to contexts differing in these aspects. Still, this scenario is much closer to typical educational conditions than commonly cited prior simulation studies. Such prior studies have even considered other types of models than latent transition analysis (e.g., latent class analysis and growth mixture modeling, Nylund et al., 2007), or scenarios that are further away from typical educational research regarding sample size and measurement design (e.g., intensive longitudinal studies with many more data points; Bacci et al., 2014; Gudicha et al., 2014). Thus, overall, our study appears to be the simulation study currently most closely adapted to realistic educational contexts.

We have taken a step towards generalizability by providing a few alternative conditions in our robustness check. These results are generally in line with our main findings, although they also indicate some deviations that might be further explored in future research. For example, in a scenario with more linear/less overlapping profiles, as they are commonly found in research on motivation (e.g., Costache et al., 2021) and with four indicator variables, the fit indices showed 
generally better performance. A deviation from our main findings was that under good conditions (e.g., $N=1000$ ) and with only two measurement points, the $B I C$ and $C A I C$ (particularly the $B I C$ ) worked better than in the other conditions. Apparently, under these conditions the higher penalty terms of these two indices work out better. From this finding, we derive the hypothesis for future research that in an LTA with only two measurement points and a sample size of at least $N=500$, the $B I C$ is the most precise index (although note that even under these conditions, its precision was just at about 50\%).

Finally, apart from the number of profiles, it would be informative to conduct simulation studies that inform about the impact of sample sizes and additional conditions on the amount of bias in the estimated profile and transition parameters. Conducting such simulation studies in mixture modeling is, however, aggravated by an issue specific to this kind of model that is called label switching (Stephens, 2000). This occurs when across different simulation runs, the profiles switch their numeric labels, such that it cannot be told which profile is which in the simulated datasets. A potential solution to this issue has been proposed that might be harnessed in future research (Tueller et al., 2011).

\section{Implications for Educational Research}

Our results show that the $B I C$, typically believed to be a superior fit index for mixture models (as indicated for example by the very large number of papers employing this index referring to Nylund et al., 2007), performs poorly in applications of latent transition analysis to typical educational contexts. The $B I C$ almost constantly underfits, meaning that it points towards models with too few learner profiles. This has direct implications for published research and for future research. For published research applying latent transition analysis in education, this finding indicates that reliance on the $B I C$ for model selection might have led researchers to 
selecting models with too few profiles. We suggest researchers to re-evalute their model selection strategies and, in case original data are still available, to re-investigate their original analyses and check whether they might have been overlooked profiles that the $A I C, A I C 3$, or the $a B I C$ would have detected.

In the application of LTA in educational research, we suggest putting less emphasis in model selection on the $B I C$ and more on the $A I C, a B I C$, and $A I C 3$. These indices work rather well with sample sizes of 200 individuals or more. We suggest researchers to rely more strongly on these fit indices when selecting which model to further examine and report. Optimally, this would include looking into a combination of at least two indices, for example either the $A I C$ for up to $N=200$ or the $A I C 3$ from $N=500$ together with the $a B I C$. The robustness checks indicated that in cases where more linear profiles with less overlapping patterns across indicator variables are plausible or empirically found, all fit indices work better. In addition, when only one or two measurement points are analyzed, the $A I C$ tends to overfit; in such cases, we suggest researchers to rely more strongly on the $a B I C$, because it seems to find a better balance of complexity and fit in such scenarios. To which extent these additional results hold across further conditions should be the concern of future research. To sum up our recommendations, in Table 3 we provide an overview of guidelines for selecting appropriate fit indices among based on our findings.

\section{Table 3}

Derived Guidelines for Fit Indices for Latent Transition Analysis

\begin{tabular}{llc}
\hline & $\begin{array}{l}\text { Moderate } \\
\text { sample } \\
(\mathrm{N}=100-200)\end{array}$ & $\begin{array}{c}\text { Large sample } \\
(\mathrm{N}=500-1000)\end{array}$ \\
\hline $\begin{array}{l}\text { Moderate } \\
\text { entropy } \\
(<=.82)\end{array}$ & AIC \& aBIC & aBIC \& AIC3 \\
$\begin{array}{l}\text { High entropy } \\
(>=.87)\end{array}$ & AIC \& aBIC & AIC $^{\text {a } ~ A I C 3 ~}$ \\
\hline
\end{tabular}


ander atrrition, the weight should be shifted more towards the AIC.

Coming back to the applied example by Flaig et al. (2018), we can now see how our results would suggest solving the issue of how many latent profiles to model. At a sample size of $N=137$, entropy of $.83-.86$ across time points, and dropout of $17 \%$, the $A I C$ and adjusted $B I C$ pointed towards five profiles, the $A I C 3$ towards three or four, and the BIC and $C A I C$ towards five profiles. Given our guidelines visible from Table 3, we would suggest relying more on the $A I C$ and adjusted $B I C$ than on the regular $B I C$ and the $C A I C$, and perhaps modestly on the $A I C 3$. Our results point towards the five profile-solution for their study and our findings regarding the $B I C$ indicate that this index should not have been used to justify ignoring the five profile-solution. The five profile solution still could have been dropped for theoretical reasons that our study cannot inform about, such as the added theoretical information value of the fifth profile, or the amount of learners that would have been accounted to this additional profile. This example demonstrates that shifting the weight away from the $B I C$ in realistic data situations can affect researchers' decisions.

Readers might wonder how they should obtain the different fit indices, since not all of these are provided in typical software packages that estimate latent transition analyses. We have programmed a simple online tool that researchers can freely use to compute the different fit indices. The tool is available under https://peter1328.shinyapps.io/FitApp/ and readers can use this app to compute all of these fit indices with a simple click. As a final point, we stress that prior empirical findings and established theories, as well as the researcher's expertise should be incorporated into the model selection process. The combination of the highlighted fit indices with such information will help to prevent overlooking profiles that might be theoretically or 
empirically relevant, while also avoiding the extraction of profiles that might be spurious or not deliver informative insights into individual differences between learners. 


\section{References}

Aho, K., Derryberry, D., \& Peterson, T. (2014). Model selection for ecologists: The worldviews of AIC and BIC. Ecology, 95(3), 631-636. https://doi.org/10.1890/13-1452.1

Bacci, S., Pandolfi, S., \& Pennoni, F. (2014). A comparison of some criteria for states selection in the latent Markov model for longitudinal data. Advances in Data Analysis and Classification, 8(2), 125-145. https://doi.org/10.1007/s11634-013-0154-2

Chen, Q., Luo, W., Palardy, G. J., Glaman, R., \& McEnturff, A. (2017). The Efficacy of Common Fit Indices for Enumerating Classes in Growth Mixture Models When Nested Data Structure Is Ignored: A Monte Carlo Study. SAGE Open, 7(1), 2158244017700459. https://doi.org/10.1177/2158244017700459

Collins, L. M., \& Lanza, S. T. (2010). Latent Class and Latent Transition Analysis: With Applications in the Social, Behavioral, and Health Sciences. John Wiley \& Sons.

Compton, D. L., Fuchs, D., Fuchs, L. S., Elleman, A. M., \& Gilbert, J. K. (2008). Tracking children who fly below the radar: Latent transition modeling of students with lateemerging reading disability. Learning and Individual Differences, 18(3), 329-337. https://doi.org/10.1016/j.lindif.2008.04.003

Costache, O., Edelsbrunner, P. A., Becker, E., Sticca, F., Staub, F. C., \& Götz, T. (2021). Who loses motivation and who keeps it up? Investigating student and teacher Factors for student motivational development across multiple subjects. PsyArXiv preprint available from psyarxiv.com

Dziak, J. J., Coffman, D. L., Lanza, S. T., Li, R., \& Jermiin, L. S. (2020). Sensitivity and specificity of information criteria. Briefings in Bioinformatics, 21(2), 553-565. https://doi.org/10.1093/bib/bbz016 
Edelsbrunner, P. A. (2017). Domain-general and domain-specific scientific thinking in childhood: measurement and educational interplay (Doctoral dissertation, ETH Zurich).

Edelsbrunner, P. A., Schalk, L., Schumacher, R., \& Stern, E. (2015). Pathways of con-ceptual change: Investigating the influence of experimentation skills on conceptual knowledge development in early science education. Proceedings of the 37th Annual Conference of the Cognitive Science Society, 1(1), 620-625. https://www.researchcollection.ethz.ch/handle/20.500.11850/108017

Edelsbrunner, P. A., Schalk, L., Schumacher, R., \& Stern, E. (2018). Variable control and conceptual change: A large-scale quantitative study in elementary school. Learning and Individual Differences, 66, 38-53. https://doi.org/10.1016/j.lindif.2018.02.003

Flaig, M., Simonsmeier, B. A., Mayer, A.-K., Rosman, T., Gorges, J., \& Schneider, M. (2018). Reprint of "Conceptual change and knowledge integration as learning processes in higher education: A latent transition analysis.” Learning and Individual Differences, 66, 92-104. https://doi.org/10.1016/j.lindif.2018.07.001

Fonseca, J. R. (2018). Information Criteria’s Performance in Finite Mixture Models with Mixed Features. Journal of Scientific Research and Reports, 21, 1-9. https://doi.org/10.9734/jsrr/2018/v21i121826

Franzen, P., Arens, A. K., Greiff, S., \& Niepel, C. (2022). Student profiles of self-concept and interest in four domains: A latent transition analysis. Learning and Individual Differences, 95(4):102139. https://doi.org/10.1016/j.lindif.2022.102139

Fryer, L. K. (2017). (Latent) transitions to learning at university: A latent profile transition analysis of first-year Japanese students. Higher Education, 73(3), 519-537. https://doi.org/10.1007/s10734-016-0094-9 
Gaspard, H., Wille, E., Wormington, S. V., \& Hulleman, C. S. (2019). How are upper secondary school students' expectancy-value profiles associated with achievement and university STEM major? A cross-domain comparison. Contemporary Educational Psychology, 58, 149-162. https://doi.org/10.1016/j.cedpsych.2019.02.005

Gillet, N., Morin, A. J., \& Reeve, J. (2017). Stability, change, and implications of students' motivation profiles: A latent transition analysis. Contemporary Educational Psychology, 51, 222-239. https://doi.org/10.1016/j.cedpsych.2017.08.006

Grimm, A., Edelsbrunner, P. A:, \& Möller, K. (2021). Accommodating Heterogeneity: The Interaction of Instructional Scaffolding with Student Preconditions in the Learning of Hypothesis-Based Reasoning. Preprint available from psyarxiv.com/sn9c3

Gudicha, D. W., Schmittmann, V. D., Tekle, F. B., \& Vermunt, J. K. (2016). Power analysis for the likelihood-ratio test in latent Markov models: shortcutting the bootstrap p-value-based method. Multivariate Behavioral Research, 51(5), 649-660. https://doi.org/10.1080/00273171.2016.1203280

Gudicha, D. W., Schmittmann, V. D., \& Vermunt, J. K. (2016). Power computation for likelihood ratio tests for the transition parameters in latent Markov models. Structural Equation Modeling, 23(2), 234-245. https://doi.org/10.1080/10705511.2015.1014040

Hallquist, M. N., \& Wiley, J. F. (2018). MplusAutomation: an R package for facilitating largescale latent variable analyses in M plus. Structural equation modeling: a multidisciplinary journal, 25(4), 621-638. https://doi.org/10.1080/10705511.2017.1402334

Hardy, I., Jonen, A., Möller, K., \& Stern, E. (2006). Effects of instructional support within constructivist learning environments for elementary school students' understanding of 
“floating and sinking." Journal of Educational Psychology, 98(2), 307-326. https://doi.org/10.1037/0022-0663.98.2.307

Harring, J. R., \& Hodis, F. A. (2016). Mixture modeling: Applications in educational psychology. Educational Psychologist, 51(3-4), 354-367. https://doi.org/10.1080/00461520.2016.1207176

Hickendorff, M., Edelsbrunner, P. A., McMullen, J., Schneider, M., \& Trezise, K. (2018). Informative tools for characterizing individual differences in learning: Latent class, latent profile, and latent transition analysis. Learning and Individual Differences, 66, 4-15. https://doi.org/10.1016/j.lindif.2017.11.001

Hulleman, C. S., \& Cordray, D. S. (2009). Moving From the Lab to the Field: The Role of Fidelity and Achieved Relative Intervention Strength. Journal of Research on Educational Effectiveness, 2(1), 88-110. https://doi.org/10.1080/19345740802539325

Kainulainen, M., McMullen, J., \& Lehtinen, E. (2016). Early Developmental Trajectories Toward Concepts of Rational Numbers. Cognition and Instruction, 35(1), 4-19. https://doi.org/10.1080/07370008.2016.1251287

Lin, T. H., \& Dayton, C. M. (1997). Model Selection Information Criteria for Non-Nested Latent Class Models. Journal of Educational and Behavioral Statistics, 22(3), 249-264. https://doi.org/10.3102/10769986022003249

McMullen, J., Laakkonen, E., Hannula-Sormunen, M., \& Lehtinen, E. (2015). Modeling the developmental trajectories of rational number concept(s). Learning and Instruction, 37, 14-20. https://doi.org/10.1016/j.1earninstruc.2013.12.004 
Mellard, D. F., Woods, K. L., \& Lee, J. H. (2016). Literacy profiles of at-risk young adults enrolled in career and technical education. Journal of Research in Reading, 39(1), 88108. https://doi.org/10.1111/1467-9817.12034

Morgan, G. B. (2015). Mixed Mode Latent Class Analysis: An Examination of Fit Index Performance for Classification. Structural Equation Modeling: A Multidisciplinary Journal, 22(1), 76-86. https://doi.org/10.1080/10705511.2014.935751

Muthén, L. K., \& Muthén, B. O. (1998-2011). Mplus User's Guide. Sixth Edition. Los Angeles, CA: Muthén \& Muthén.

Nelson, M. C., Cordray, D. S., Hulleman, C. S., Darrow, C. L., \& Sommer, E. C. (2012). A Procedure for Assessing Intervention Fidelity in Experiments Testing Educational and Behavioral Interventions. The Journal of Behavioral Health Services \& Research, 39(4), 374-396. https://doi.org/10.1007/s11414-012-9295-X

Nylund, K. L., Asparouhov, T., \& Muthén, B. O. (2007). Deciding on the Number of Classes in Latent Class Analysis and Growth Mixture Modeling: A Monte Carlo Simulation Study. Structural Equation Modeling: A Multidisciplinary Journal, 14(4), 535-569. https://doi.org/10.1080/10705510701575396

Peugh, J., \& Fan, X. (2013). Modeling Unobserved Heterogeneity Using Latent Profile Analysis: A Monte Carlo Simulation. Structural Equation Modeling: A Multidisciplinary Journal, 20(4), 616-639. https://doi.org/10.1080/10705511.2013.824780

R Core Team (2017). R: A language and environment for statistical computing. R Foundation for Statistical Computing, Vienna, Austria. 
Schneider, M., \& Hardy, I. (2013). Profiles of inconsistent knowledge in children's pathways of conceptual change. Developmental Psychology, 49(9), 1639-1649. https://doi.org/10.1037/a0030976

Schwichow, M., Osterhaus, C., Edelsbrunner, P. A. (2020). The Relationship between the Control-of-Variables Strategy and Science Content Knowledge in Secondary School. Contemporary Educational Psychology, 63, 101923. https://doi.org/10.1016/j.cedpsych.2020.101923

Stephens, M. (2000). Dealing with label switching in mixture models. Journal of the Royal Statistical Society: Series B (Statistical Methodology), 62(4), 795-809. https://doi.org/10.1111/1467-9868.00265

Straatemeier, M., van der Maas, H. L., \& Jansen, B. R. (2008). Children's knowledge of the earth: A new methodological and statistical approach. Journal of Experimental Child Psychology, 100(4), 276-296. https://doi.org/10.1016/j.jecp.2008.03.004

Swanson, H. L. (2012). Cognitive profile of adolescents with math disabilities: Are the profiles different from those with reading disabilities? Child Neuropsychology, 18(2), 125-143. https://doi.org/10.1080/09297049.2011.589377

Tein, J.-Y., Coxe, S., \& Cham, H. (2013). Statistical Power to Detect the Correct Number of Classes in Latent Profile Analysis. Structural Equation Modeling: A Multidisciplinary Journal, 20(4), 640-657. https://doi.org/10.1080/10705511.2013.824781

Tolvanen, A. (2007). Latent growth mixture modeling: A simulation study (No. 111). University of Jyväskylä.

Tueller, S. J., Drotar, S., \& Lubke, G. H. (2011). Addressing the Problem of Switched Class Labels in Latent Variable Mixture Model Simulation Studies. Structural Equation 
Modeling: A Multidisciplinary Journal, 18(1), 110-131. https://doi.org/10.1080/10705511.2011.534695

Van Der Maas, H. L., \& Straatemeier, M. (2008). How to detect cognitive strategies:

commentary on 'Differentiation and integration: guiding principles for analyzing cognitive change'. Developmental science, 11(4), 449-453.

https://doi.org/10.1111/j.1467-7687.2008.00690.x

Vonesh, E., Chinchilli, V. (1997). Linear and Nonlinear Models for the Analysis of Repeated Measurements. Boca Raton: CRC Press.

Wickham, H., Averick, M., Bryan, J., Chang, W., McGowan, L. D. A., François, R., ... \& Yutani, H. (2019). Welcome to the Tidyverse. Journal of Open Source Software, 4(43), 1686. https://doi.org/10.21105/joss.01686 


\section{A Simulation Study on Latent Transition Analysis for Examining Profiles and Trajectories in Education: Recommendations for Fit Statistics}

\section{Supplementary materials}

S1 Description of the Entropy Index....................................... S2

S2 Extended Rationales for the Simulated Scenarios.......................... S2

S3 Details Regarding the Simulated Models................................ S7

S4 Detailed Results Regarding Over- and Underfitting for the Different Fit Indices..p. S9

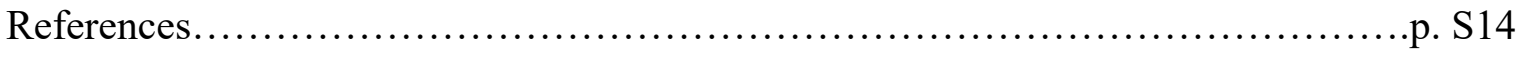




\section{S1 Description of the Entropy Index}

The equation for entropy as given for example in Collins \& Lanza (2010; pp. 74-75) that has been proposed by Ramaswamy et al. (1993) is

$$
E=\frac{1-\sum_{i=i}^{n} \sum_{c=1}^{C}-p_{i c} \log p_{i c}}{n \log C}
$$

Where $p_{\text {ic }}$ is an individual's $i$ posterior probability $p$ to belong to transition pattern $c$, given their observed vectors of mean values on the indicator variables at all time points. Log refers to the natural logarithm. Thus, the closer each individual's posterior probability to be in one transition pattern is to one, the higher entropy will be, although there might be multiple individuals with high classification uncertainty and entropy can still be high across all individuals.

\section{S2 Extended Rationales for the Simulated Scenarios}

The simulated standard scenario and central simulation conditions were designed based on prior studies that have applied latent transition analysis to educational research up to December 2018. To identify such studies, we conducted a literature search in GoogleScholar and Scopus using the search string "latent transition" OR "hidden markov" OR "latent markov" AND education OR learn*. The results encompass eight hits that referred to studies published in peer-reviewed journals that were concerned with substantive questions related to education (which were our selection criteria). The characteristics of these studies relevant to our simulation setup are presented in Table S1. As can be seen from this table, most studies applying latent transition analysis had been concerned with modeling learners' development of conceptual knowledge. Typically, such a study would involve an intervention between the first and second measurements. Only in two cases, studies examined learners across measurement waves without 
specific intervention. All studies modeled learners' profiles based on continuous indicator variables, typically representing different kinds of knowledge (e.g., scores representing misconceptions and scientifically correct concepts, or different kinds of typical concepts within a topic). Typical sample sizes varied between slightly above $N=100$, and than $N=1000$. Reported dropout across measurement points varied between $0 \%$ and $30 \%$. On average, the studies reported development across three measurement points and used three indicator variables to represent learners' development. Concerning the typical results of these studies, they would typically opt for a solution with four learner profiles, yielding entropy values between slightly above .70 and almost 1.00 , and reporting that most learners showed between three and eight transition patterns with very high probabilities.

Based on these typical characteristics, we, first of all, decided to model continuous indicator variables, since all studies had done that. We simulated three indicator variables at three time points, assuming that an intervention would take place between the first and the second measurement point, since that was the typical scenario in those studies. In our standard scenario, we furthermore modelled four latent profiles, since that was the typical number of profiles typically reported in the studies. What is not visible from Table S1 but can be seen from the original articles is that in all but one study (Compton et al., 2008), the researchers found highly non-linear/overlapping profile solutions, meaning that there were not just level differences between profile patterns, but also shape differences (see Marsh et al., 2009). We therefore also decided to model profiles with rather pronounced shape differences and thus overlapping patterns.

In our main simulations, we decided to stick to this scenario regarding the simulated variables, profiles, and transitions, and to focus on varying design factors across simulations 
conditions that would not change the substantive interpretation of this scenario (i.e., keeping the profiles and transitions, the two defining sets of parameters in the model, always the same). Thus, we decided to vary entropy, sample size, and sample dropout, which are three variables that do not change the substantial interpretation of the profiles and transition patterns. In these main simulation conditions, we varied these three factors to represent most of the variation found in these three study characteristics according to Table S1. However, at the same time we did not simulate the full range of conditions that appeared of relatively little information value for future researchers applying latent transition analysis. Specifically, we decided to cover sample sizes at the lower bound found in the literature (about $N=100$ ) quite well, but we did not simulate data with more than $N=1000$ participants for two reasons. First, there was only one study having a much larger sample available (Edelsbrunner et al., 2018, with more than 1800 participants), such that this was not a common case but we considered it rather an outlier. Second, our results appeared stable already with $N=1000$, such that they remained rather similar to those obtained with $N=500$ with a few exceptions. Therefore, $N=1000$ was the largest sample size we encompassed in this factor. Regarding entropy, we decided for a low entropy-condition with $E=$ 73, which was in the range of the two studies with the lowest empirically estimated entropy values. For medium and high entropy values we decided for .82 and .87 , respectively. Whereas this does not cover the very high entropy values found in two studies in Table S1, we decided not to go so high. The reason is that in the two studies in which entropy was estimated at such high values, these results were not obtained on a latent transition analysis, but on latent profile analyses that the researchers conducted on individual time points before estimating the full transition analysis across all time points. Based on pilot simulations, we realized that such high entropy values on individual time points are usually linked to entropies of somewhere about .80 
to .85 or a bit above in the resulting full latent transition analysis. Thus, we decided to go with a value of .87 for high entropy that we would have assumed to result from the full latent transition analysis in these studies.

The reason to select dropout (i.e., missing data) as the third simulated factor was that this is an issue that many researchers are concerned with, as Table S1 confirms. Note that in one of the studies indicating $0 \%$ of missing data (Edelsbrunner et al., 2018) the authors indicated that they did only use the data of participants who had complete data across their two measurement points. Thus, even in that study missing data would have been present, but we do not know its precise extent. 


\section{Table S1}

Characteristics of Studies Applying Latent Transition Analysis in Educational Research up to December 2019

\begin{tabular}{|c|c|c|c|c|c|c|c|c|c|c|}
\hline Reference & Topic & Intervention & $\begin{array}{l}\text { Sample } \\
\text { size T1 }\end{array}$ & $\begin{array}{l}\text { Overall } \\
\text { dropout }\end{array}$ & $\begin{array}{l}\text { Time } \\
\text { points }\end{array}$ & $\begin{array}{l}\text { Indicator } \\
\text { variables }\end{array}$ & $\begin{array}{c}\text { Indicator } \\
\text { scale }\end{array}$ & $\begin{array}{c}\text { Resulting } \\
\text { classes/profiles }\end{array}$ & Entropy & $\begin{array}{l}\text { Frequent } \\
\text { transitions }\end{array}$ \\
\hline $\begin{array}{l}\text { Compton et } \\
\text { al. (2008) }\end{array}$ & Reading & yes & 252 & $30 \%$ & 2 & 3 & Interval & 2 & NA & 3 \\
\hline $\begin{array}{l}\text { Edelsbrunner } \\
\text { et al. (2018) }\end{array}$ & $\begin{array}{l}\text { Conceptual } \\
\text { Knowledge }\end{array}$ & yes & 1809 & $0 \%$ & 2 & 3 & Interval & 4,6 & .72 & NA \\
\hline $\begin{array}{l}\text { Flaig et al. } \\
\text { (2018) }\end{array}$ & $\begin{array}{l}\text { Conceptual } \\
\text { Knowledge }\end{array}$ & no & 137 & $17 \%$ & 4 & 3 & Interval & 4 & $.83-.86^{\mathrm{a}}$ & 6 \\
\hline Fryer (2017) & $\begin{array}{l}\text { Learning } \\
\text { Strategies }\end{array}$ & no & 920 & $<3 \%$ & 2 & 4 & Interval & 3 & $.79 / .80^{a}$ & NA \\
\hline $\begin{array}{l}\text { Gillet et al. } \\
\text { (2017) }\end{array}$ & Motivation & no & 504 & & 2 & & Interval & 6 & .84 & 6 \\
\hline $\begin{array}{l}\text { Kainulainen } \\
\text { et al. (2017) }\end{array}$ & $\begin{array}{l}\text { Conceptual } \\
\text { Knowledge }\end{array}$ & yes & 251 & $20 \%$ & 3 & 4 & Interval & 5 & $.97-.98^{\mathrm{a}}$ & 8 \\
\hline $\begin{array}{l}\text { McMullen et } \\
\text { al. }(2015)\end{array}$ & $\begin{array}{l}\text { Conceptual } \\
\text { Knowledge }\end{array}$ & yes & 251 & $12 \%$ & 3 & 3 & Interval & 4 & $.92-.95^{\mathrm{a}}$ & 6 \\
\hline $\begin{array}{l}\text { Schneider \& } \\
\text { Hardy (2013) }\end{array}$ & $\begin{array}{l}\text { Conceptual } \\
\text { Knowledge }\end{array}$ & yes & 161 & $0 \%$ & 3 & 3 & Interval & 5 & .73 & 7 \\
\hline
\end{tabular}

Note. ${ }^{\mathrm{a}}$ Multiple entropy values are indicated because in the respective studies the researchers obtained entropy estimates not from a latent transition analysis, but form latent profile analyses at the individual time points; indicated values are the lowest and highest ones reported. Entropy value from Schneider \& Hardy obtained from original data set. Frequent transitions indicates number of transitions paths that were taken by most students (usually by at least $85 \%$ ), indicating homogeneity of developmental patterns. 


\section{S3 Details Regarding the Simulated Models}

The central model that we have simulated is presented in Figure A1. The model defines three (latent) state-variables, S1, S2, and S3 representing learners' profiles at the three measurement points, $\mathrm{t} 1, \mathrm{t} 2$, and $\mathrm{t} 3$. The profiles at each measurement point are defined through patterns of mean values and variances across the three (observed) indicator variables $\mathrm{x} 1, \mathrm{x} 2$, and $\mathrm{x} 3$. The profiles have the same mean and variance parameters at each measurement point. Learners' development is captured in a Markov chain-structure represented in the regression paths S1 -> S2 and S2 -> S3. These regressions are defined via transition probabilities that indicate the probabilities of staying within the same profile, or transitioning into a different profile, between two measurement points.

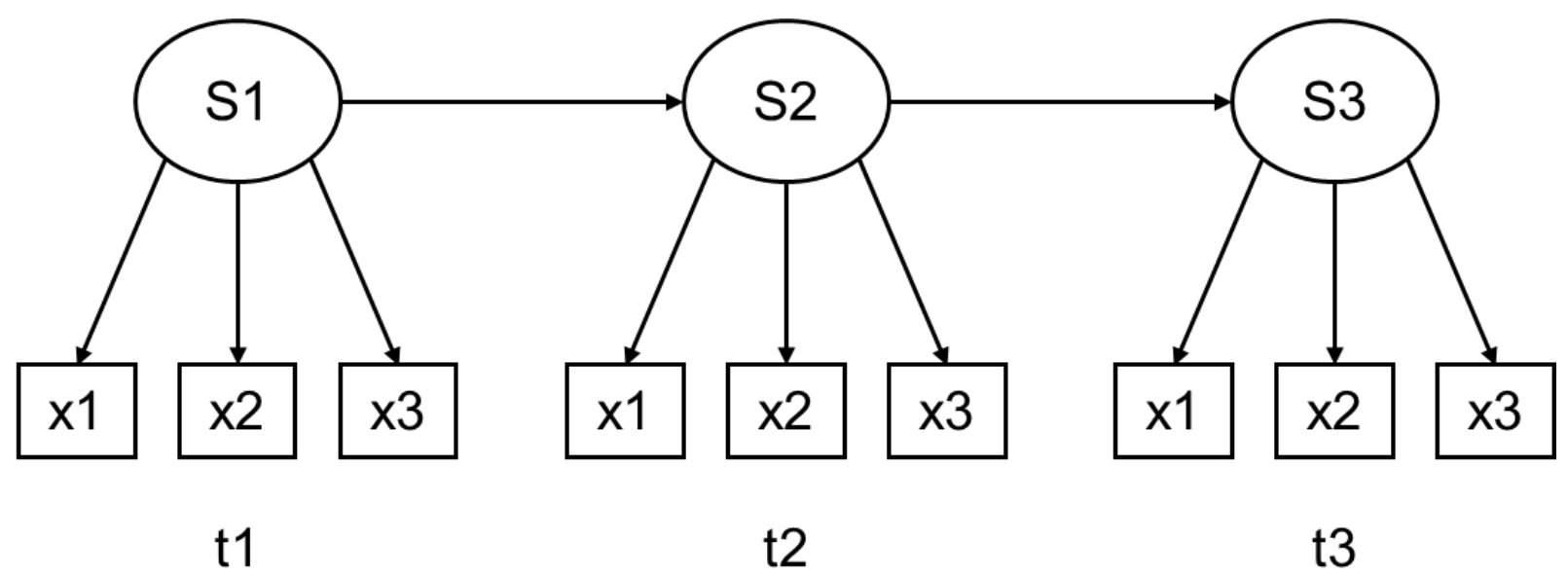

An individuals' observed pattern of means on the three indicator variables is given by the vector $\vec{x}_{\mathrm{t}}=(\mathrm{x} 1, \mathrm{x} 2, \mathrm{x} 3)$ at time point $t$.

The four states/profiles at each measurement point are measurement invariant over time (i.e., they are defined by the same patterns of means and variances, with zero off-diagonal covariances) and are given by $S \in\{$ Profile1, Profile2, Profile3, Profile4\}.

The probabilities to transition from one profile into another between the first and the second measurement point are defined trough the transition matrix 


\begin{tabular}{|c|c|c|c|}
\hline & $S 1$ & $S 2 \quad S 3$ & $S 4$ \\
\hline$S 1$ & $/ p 11$ & $p 12 p 13$ & $p 14$ \\
\hline $\begin{array}{r}S 2 \\
P_{1 \rightarrow 2} S 3\end{array}$ & & $\because$ & \\
\hline$S 4$ & $\backslash p 41$ & $\cdots$ & $p 44$ \\
\hline
\end{tabular}

With the condition that the probabilities in each row must sum to $1: \sum_{j=1}^{4} S_{i j}=1$

For the transitions from $\mathrm{t} 2$ to $\mathrm{t} 3$, we have a matrix of the same structure but with different parameters for the transition probabilities.

For each profile $S_{\mathrm{i}}$, we have $\vec{x} \mid S_{j} \sim \operatorname{MvN}\left(\vec{\mu}_{t}, \Sigma_{j}\right)$ with the variance-covariance matrix having zero off-diagonal elements, such that there are no covariances within states, implying local independence.

Given the Markov property, the joint likelihood for an individual is then given by

$$
\begin{gathered}
p\left(\vec{x}_{1}, \vec{x}_{2}, \vec{x}_{3}, S_{1}, S_{2}, S_{3}\right)=p\left(\vec{x}_{1} \mid S_{1}\right) p\left(S_{1}\right) p\left(\vec{x}_{2} \mid S_{2}\right) p\left(S_{2} \mid S_{1}\right) p\left(\vec{x}_{3} \mid S_{3}\right) p\left(S_{3} \mid S_{2}\right)= \\
p\left(\vec{x}_{3} \mid S_{3}\right) p\left(\vec{x}_{2} \mid S_{2}\right) p\left(\vec{x}_{1} \mid S_{1}\right) p\left(S_{3} \mid S_{2}\right) p\left(S_{2} \mid S_{1}\right) p\left(S_{1}\right)
\end{gathered}
$$




\section{S4 Detailed Results Regarding Over- and Underfitting for the Different Fit Indices}

\section{Figure S2}

\section{Detailed Results for the AIC}

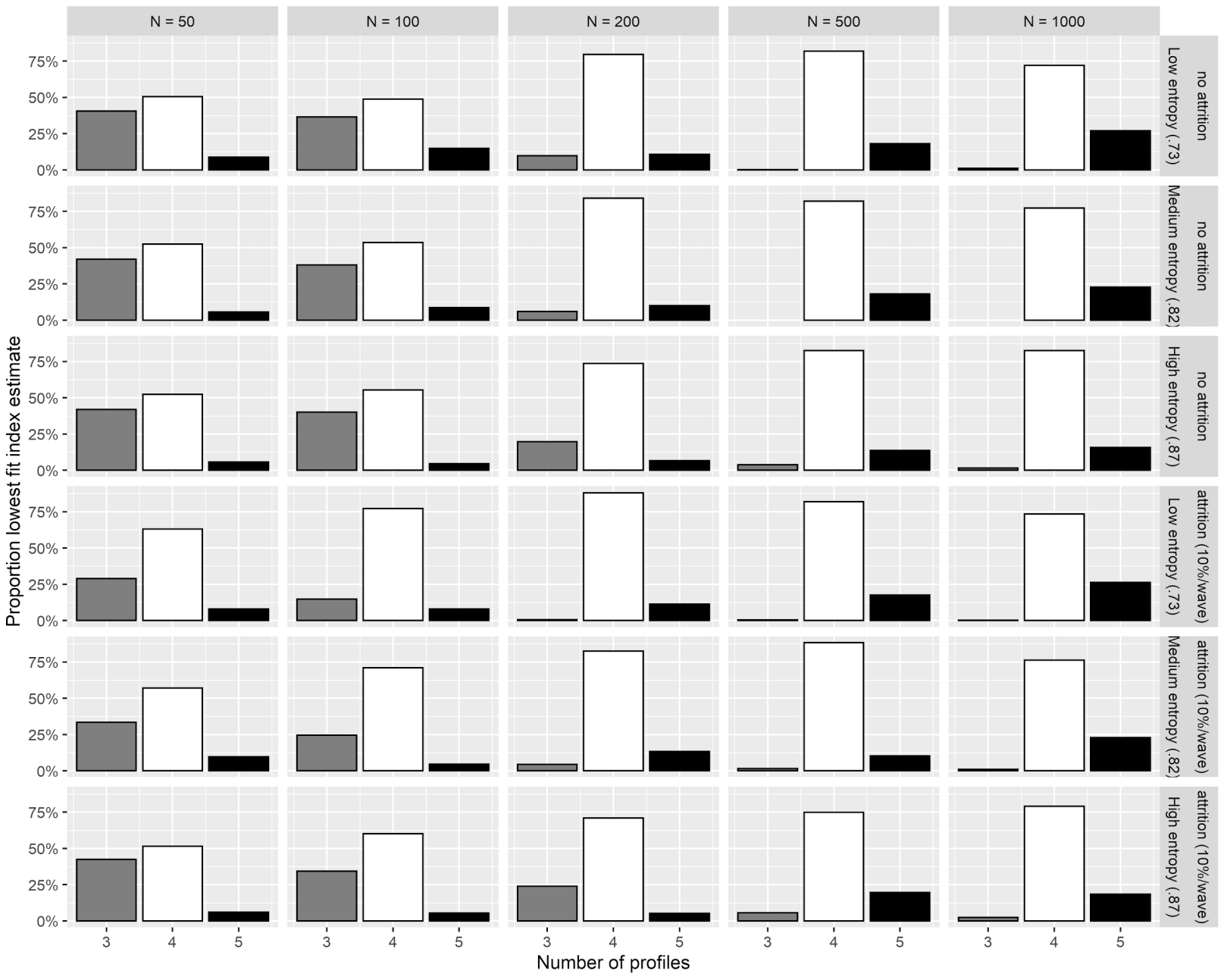

Note. The image indicates in percentage (y-axis) how often the lowest AIC occurred under the models with different numbers of estimated latent profiles (x-axis: 3 profiles/underestimation in grey, 4 profiles/correct model in white, 5 profiles/overestimation in black). 


\section{Figure S3}

Detailed Results for the BIC

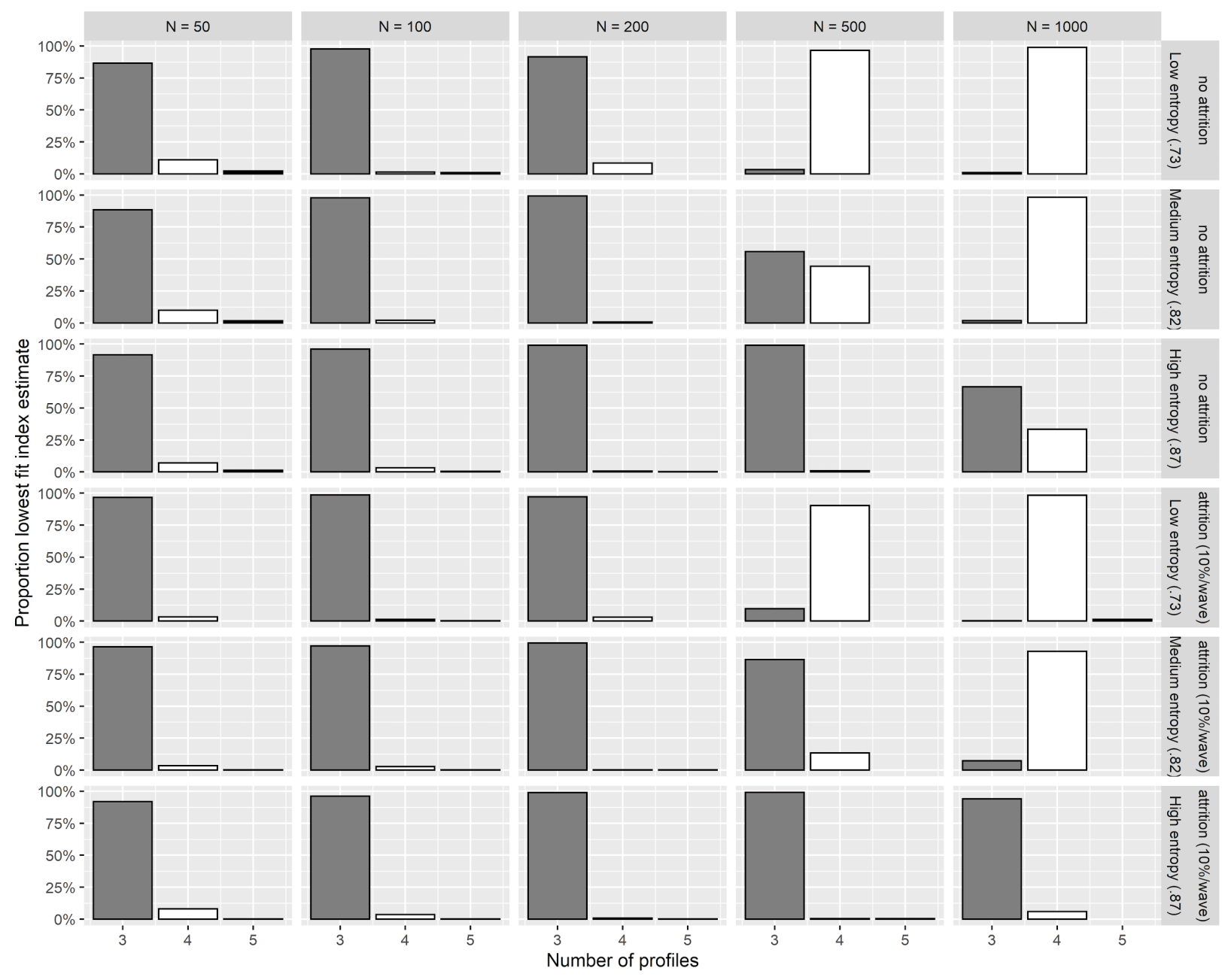

Note. The image indicates in percentages (y-axis) how often the lowest BIC occurred under the models with different numbers of estimated latent profiles (x-axis: 3 profiles/underestimation in grey, 4 profiles/correct model in white, 5 profiles/overestimation in black). 


\section{Figure S4}

Detailed Results for the aBIC

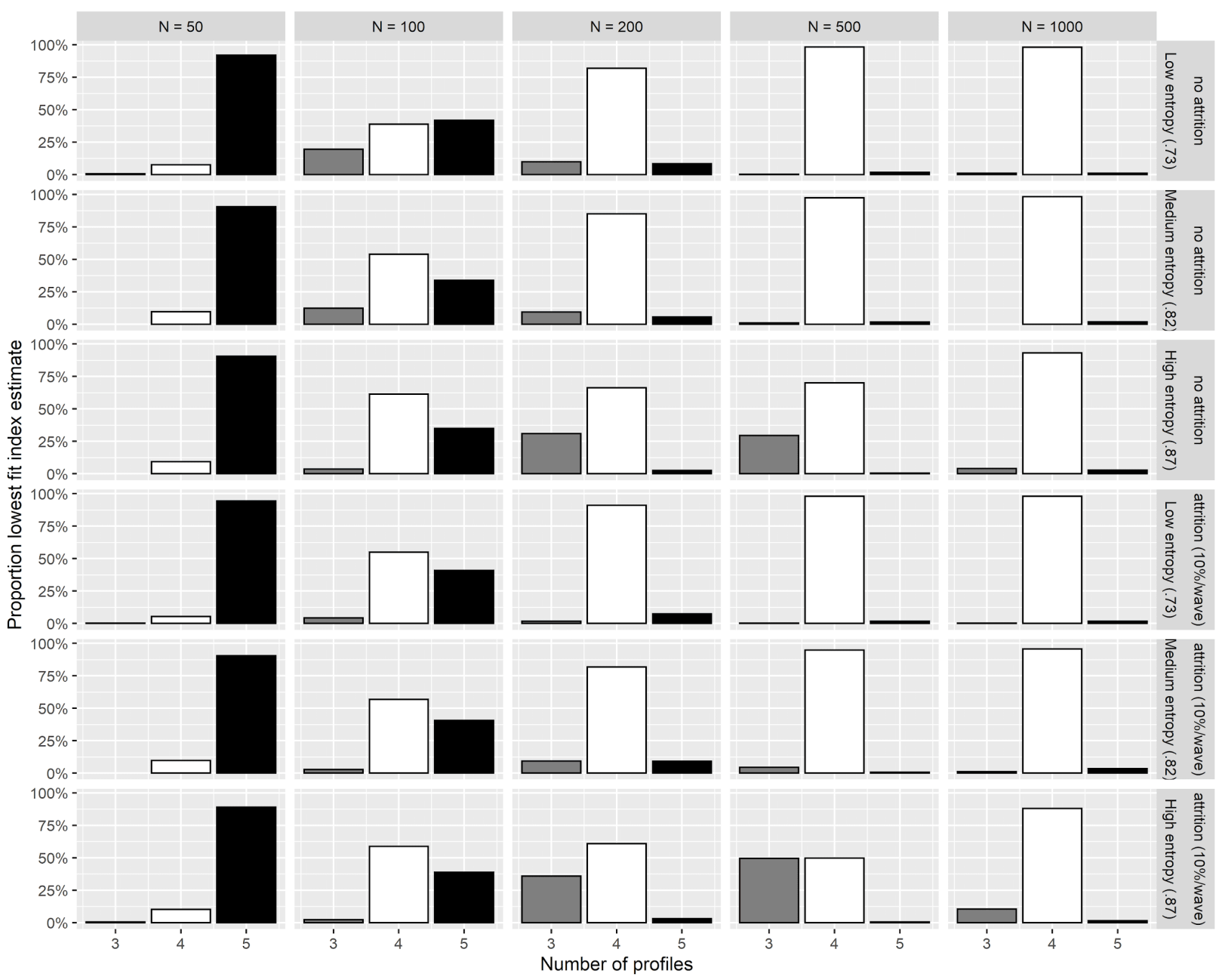

Note. The image indicates in percentages (y-axis) how often the lowest $a B I C$ occurred under the models with different numbers of estimated latent profiles (x-axis: 3 profiles/underestimation in grey, 4 profiles/correct model in white, 5 profiles/overestimation in black). 
Figure S5

Detailed Results for the AIC3

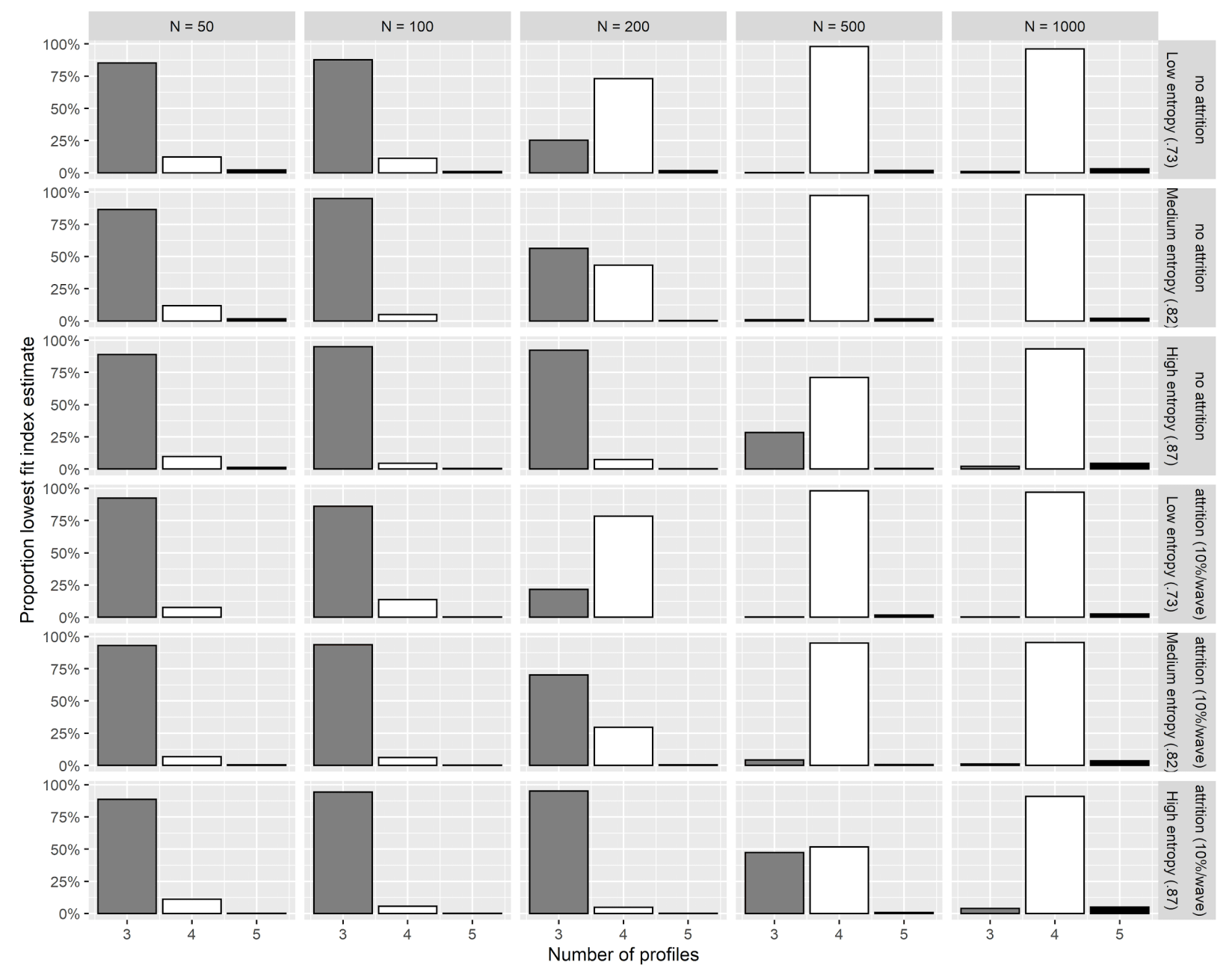

Note. The image indicates in percentage (y-axis) how often the lowest AIC 3 occurred under the models with different numbers of estimated latent profiles (x-axis: 3 profiles/underestimation in grey, 4 profiles/correct model in white, 5 profiles/overestimation in black). 


\section{Figure S6}

Detailed Results for the CAIC

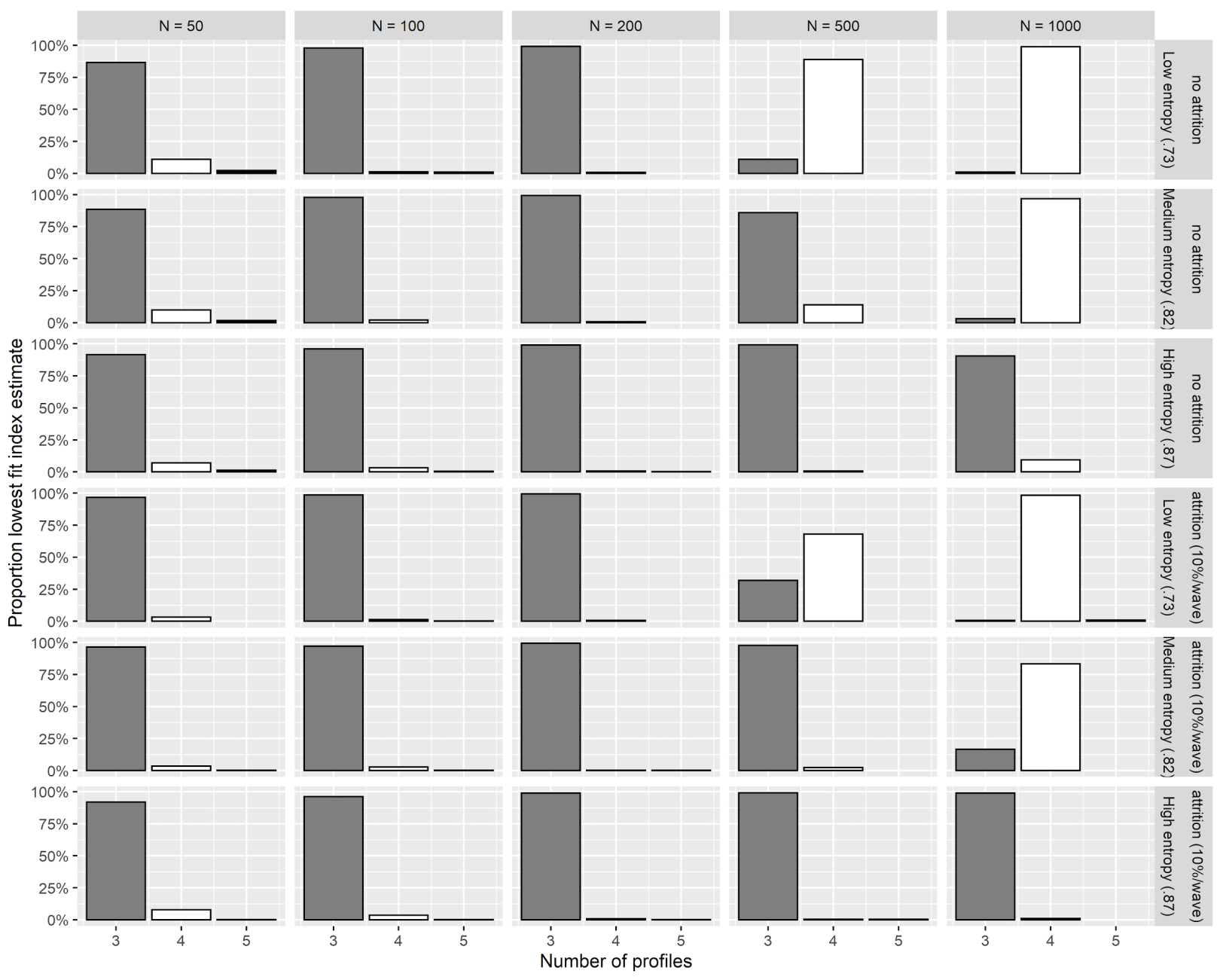

Note. The image indicates in percentage (y-axis) how often the lowest CAIC occurred under the models with different numbers of estimated latent profiles (x-axis: 3 profiles/underestimation in grey, 4 profiles/correct model in white, 5 profiles/overestimation in black). 


\section{Supplementary Materials: References}

Collins, L. M., \& Lanza, S. T. (2010). Latent Class and Latent Transition Analysis: With Applications in the Social, Behavioral, and Health Sciences. John Wiley \& Sons.

Compton, D. L., Fuchs, D., Fuchs, L. S., Elleman, A. M., \& Gilbert, J. K. (2008). Tracking children who fly below the radar: Latent transition modeling of students with late-emerging reading disability. Learning and Individual Differences, 18(3), 329-337. https://doi.org/10.1016/j.lindif.2008.04.003

Edelsbrunner, P. A., Schalk, L., Schumacher, R., \& Stern, E. (2018). Variable control and conceptual change: A large-scale quantitative study in elementary school. Learning and Individual Differences, 66, 38-53. https://doi.org/10.1016/j.lindif.2018.02.003

Flaig, M., Simonsmeier, B. A., Mayer, A.-K., Rosman, T., Gorges, J., \& Schneider, M. (2018). Reprint of "Conceptual change and knowledge integration as learning processes in higher education: A latent transition analysis.” Learning and Individual Differences, 66, 92-104. https://doi.org/10.1016/j.lindif.2018.07.001

Fryer, L. K. (2017). (Latent) transitions to learning at university: A latent profile transition analysis of first-year Japanese students. Higher Education, 73(3), 519-537. https://doi.org/10.1007/s10734$\underline{016-0094-9}$

Gillet, N., Morin, A. J. S., \& Reeve, J. (2017). Stability, change, and implications of students’ motivation profiles: A latent transition analysis. Contemporary Educational Psychology, 51, 222-239. https://doi.org/10.1016/j.cedpsych.2017.08.006

Kainulainen, M., McMullen, J., \& Lehtinen, E. (2017). Early Developmental Trajectories Toward Concepts of Rational Numbers. Cognition and Instruction, 35(1), 4-19. https://doi.org/10.1080/07370008.2016.1251287 
Marsh, H. W., Lüdtke, O., Trautwein, U., \& Morin, A. J. S. (2009). Classical Latent Profile Analysis of Academic Self-Concept Dimensions: Synergy of Person- and Variable-Centered Approaches to Theoretical Models of Self-Concept. Structural Equation Modeling: A Multidisciplinary Journal, 16(2), 191-225. https://doi.org/10.1080/10705510902751010

McMullen, J., Laakkonen, E., Hannula-Sormunen, M., \& Lehtinen, E. (2015). Modeling the developmental trajectories of rational number concept(s). Learning and Instruction, 37, 14-20. https://doi.org/10.1016/j.learninstruc.2013.12.004

Ramaswamy, V., Desarbo, W. S., Reibstein, D. J., \& Robinson, W. T. (1993). An Empirical Pooling Approach for Estimating Marketing Mix Elasticities with PIMS Data. Marketing Science, 12(1), 103-124. https://doi.org/10.1287/mksc.12.1.103

Schneider, M., \& Hardy, I. (2013). Profiles of inconsistent knowledge in children's pathways of conceptual change. Developmental Psychology, 49(9), 1639-1649.

https://doi.org/10.1037/a0030976 